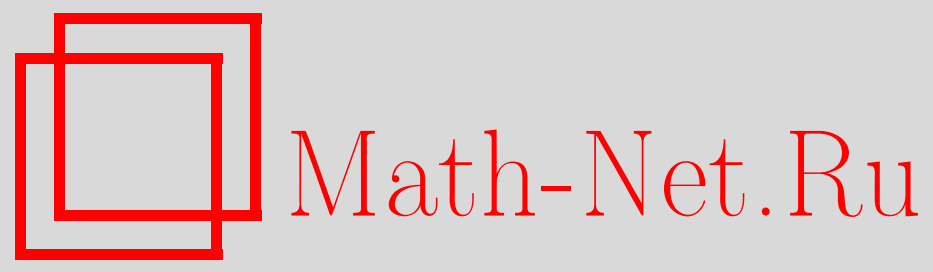

В. А. Захаров, Аппроксимация абстрактных семантик формальными моделями программ, Дискрет. матем., 1998, том 10, выпуск 4, 119-141

DOI: https://doi.org/10.4213/dm448

Использование Общероссийского математического портала Math-Net.Ru подразумевает, что вы прочитали и согласны с пользовательским соглашением http: //www . mathnet.ru/rus/agreement

Параметры загрузки:

IP : 54.196 .121 .252

26 апреля 2023 г., $10: 27: 43$

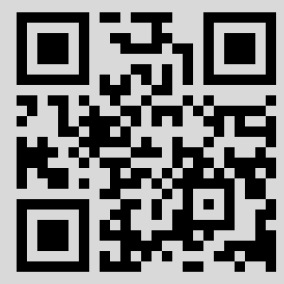




\title{
Аппроксимащия абстрактных семантик формальными моделями программ
}

\author{
(C) 1998 г. $\quad$ B. A. Захаров
}

\begin{abstract}
Решение задач анализа и оптимизации вычислительных программ в алгоритмических системах сильно осложняется нерекурсивностью функциональных свойств программ. Согласно теореме Райса в универсальном языке программирования любое свойство программ, зависящее только от вычисляемых ими функций, алгоритмически неразрешимо. Поэтому используется следующий прием. Исходная сложная семантика программ $\sigma$ заменяется более простой семантикой $\omega$ или классом семантик $\Omega$. Если выполнимость некоторого свойства программ в семантике $\omega$, или в каждой из семантик класса $\Omega$, влечет его выполнимость в семантике $\sigma$, то говорят, что семантика $\omega$, или класс $\Omega$, аппроксимирует $\sigma$ относительного заданного программного свойства. Выбрав для заданной семантики $\sigma$ подходящую аппроксимацию, в которой алгоритмически разрешимо исследуемое свойство программ, можно построить эффективную процедуру, частично разрешающую проблему анализа функциональных свойств программ в семантике $\sigma$.

Одной из центральных проблем теоретического программирования является проблема функциональной эквивалентности программ, и настоящая статья посвящена изучению вопросов аппроксимации программных семантик относительно этого свойства программ.
\end{abstract}

\section{1. Введение}

Как известно, решение задач анализа и оптимизации вычислительных программ в алгоритмических системах, обладающих достаточно богатыми выразительными возможностями, сильно осложняется нерекурсивностью функциональных свойств программ. Согласно теореме Райса [1] в универсальном языке программирования любое свойство программ, зависящее только от вычисляемых ими функций, алгоритмически неразрешимо. Для того, чтобы добиться хотя бы частичного положительного решения задач семантического анализа программ, используется следующий прием. Исходная сложная семантика программ $\sigma$ заменяется более простой семантикой $\omega$ или целым классом семантик $\Omega$. Если выполнимость некоторого свойства программ в семантике $\omega$ (или в каждой из семантик класса $\Omega$ ) влечет его выполнимость в семантике $\sigma$, то говорят, что семантика $\omega$ (класс семантик $\Omega$ ) аппроксимирует $\sigma$ относительно заданного программного свойства. Выбрав для заданной семантики $\sigma$ подходящую аппроксимацию, в которой алгоритмически разрешимо исследуемое свойство программ, можно построить эффективную процедуру, 
частично разрешающую ту или иную проблему анализа функциональных свойств программ в семантике $\sigma$. Таким образом, в рамках описанного подхода становится актуальной задача выделения для заданной программной семантики $\sigma$ всех ее аппроксимаций из класса семантик $\Omega$. Одной из центральных проблем теоретического программирования является проблема функциональной эквивалентности программ, и настоящая работа посвящена изучению некоторых вопросов аппроксимации программных семантик относительно этого свойства программ.

Мы будем рассматривать последовательностные операторные программы, семантика которых определяется интерпретацией базисных компонент программы, приписывающей операторам и логическим условиям функции и предикаты на множестве состояний памяти. Интерпретационные.семантики такого вида положены в основу операционных семантик реально существующих систем процедурного программирования. Для приближения интерпретационных семантик будут использоваться программные семантики другого типа, формальные модели программ, введенные в [2-4] и обобщающие дискретные преобразователи Глушкова-Летичевского [5]. Цель работы - установить общие требования, которым должны удовлетворять параметры формальных моделей, аппроксимирующих заданные интерпретационные семантики программ.

Статья организована следующим образом. Во втором разделе определены два типа семантик операторных программ, абстрактные семантики программ, являющиеся обобщением тотальных интерпретационных семантик $[3,4,6]$, и формальные модели программ [2-4], и формально уточнена исследовательская задача. В третьем разделе обсуждены ранее полученные результаты решения задач аппроксимации программных семантик и сравнительного анализа формальных моделей программ. В четвертом разделе внимание уделено вопросу аппроксимации абстрактных семантик формальными моделями программ. Показано, что в тех случаях, когда абстрактная семантика допускает наилучшее приближение формальной моделью программ, эта аппроксимация относится к классу правоконгруэнтных формальных моделей, и приведены примеры абстрактных семантик, имеющих наилучшую аппроксимацию в различных классах формальных моделей. В пятом разделе доказано существование абстрактных семантик, не имеющих наилучшего формального приближения, и приведены некоторые следствия этого результата. В шестом разделе рассмотрены вопросы аппроксимации тотальных семантик. Установлено, что семантики данного типа аппроксимируются полугрупповыми моделями программ $[6]$.

\section{2. Абстрактные семантики и формальные модели программ}

Мы будем исследовать функциональные свойства управляющих систем специального вида, последовательностных операторных программ, при различных интерпретациях. Синтаксическую основу программы составляет схема программы над символьным базисом, образованным парой непустых конечных множеств $S$ и $P$. Элементы множества $S$ называются операторными символами (о.с.), а элементы $P$ логическими переменными (л.п.). Слова конечной длины в алфавите $S$ будут называться операторными цепочками (о. ц.). Множество всевозможных о. ц. будем обозначать $S^{*}$, предполагая при этом, что пустая о.ц. $е$ содержится в $S^{*}$. Всякую 
начальную подцепочку $h$ о.ц. $H$ будем называть префиксом $H$. Через $|h|$ будем обозначать длину о. ц. $h$.

Схема программы над базисом $(S, P)$ представляет собой конечный ориентированный граф $\pi$, в котором особо выделены две вершины, вход и выход схемы, а все прочие вершины разбиты на два непересекающихся класса, преобразователи и распознаватели. Вход имеет единственную исходящую дугу и не имеет ведущих в него дуг. Выход не имеет исходящих дуг и может иметь произвольное число входящих дуг. Преобразователи имеют только одну исходящую дугу, и каждому из них приписан некоторый о.с. из $S$. Распознаватели имеют в точности две исходящие дуги, помеченные символами 0 и 1 , и каждому из них приписана л. п. из $P$.

Здесь надлежит отметить, что в теории схем программ понятие схема программы имеет более широкое истолкование. В качестве схем программ могут выступать различные конструкции, графовые, языковые, автоматные и другие, отражающие те или иные особенности структуры и поведения моделируемой программы. Строение схемы программ может существенно отличаться от синтаксической составляющей моделируемой ею программы (см., например, схемы Мартынюка, схемы Лаврова [7]). В тех случаях, когда программа и схема программы имеют одинаковый синтаксис, но различные семантики, говорят о синтақсически неподвижном моделировании программ схемами [8]. Такой тип моделирования программ схемами наиболее часто используется при изучении функциональной эквивалентности и эквивалентных преобразований программ [9]. Это обстоятельство позволяет автору истолковывать термин схема программы в соответствии с терминологией теории управляющих систем [10], т. е. как синтаксическую составляющую управляющей системы (в данном случае программы). Сама программа при этом определяется двумя компонентами, схемой программы, описывающей ее структуру, и семантикой программы, описывающей ее функционирование.

В основе семантик операторных программ лежат следующие допущения. Каждый о.с. $s$ из $S$ обозначает некоторое всюду определенное элементарное преобразование информационных данных, а каждая л. п. $p$ из $P$ - элементарное логическое условие (предикат) на области данных программы. Вычисление на схеме программ $\pi$ при заданных начальных данных представляет собой обход графа $\pi$, в процессе которого при прохождении через преобразователь с о.c. $s$ данные изменяются согласно закону преобразования $s$, а при прохождении через распознаватель с л. п. $p$ определяется дальнейший путь вычисления в зависимости от значения условия $p$. Вычисление начинается из входа схемы и считается успешно завершенным в случае достижения выхода схемы. Состояние данных к моменту окончания вычисления считается его результатом. В зависимости от выбора области данных и способа интерпретации о. с. и л. п. различают несколько типов семантик программ. Мы будем рассматривать программные семантики двух типов - абстрактные семантики и формальные модели программ.

Абстрактная семантика $\sigma$ определяется следующими компонентами :

- областью интерпретации $\Sigma$, элементы которой будем называть состояниями памяти ;

- множеством начальных состояний памяти $\Sigma_{0}$, где $\Sigma_{0} \in \Sigma$;

- интерпретацией $I$ элементов базиса $(S, P)$, сопоставляющей каждому операторному символу $s$ из $S$ всюду определенное отображение $I s: \Sigma \rightarrow \Sigma$ и каждой логической переменной $p$ из $P$ отображение $\operatorname{Ip}: \Sigma \rightarrow\{0,1\}$. 
Абстрактную семантику $\sigma$ такого вида условимся обозначать $\left\langle\Sigma, \Sigma_{0}, I\right\rangle$. Для произвольного состояния памяти $\xi$ из $\Sigma$ и о. ц. $h$ будем полагать

$$
I h(\xi)= \begin{cases}\xi & \text { при } h=e \\ I s_{n}\left(\ldots I s_{2}\left(I s_{1}(\xi)\right) \ldots\right) & \text { при } h=s_{1} s_{2} \ldots s_{n} .\end{cases}
$$

В случае $\Sigma_{0}=\Sigma$ семантику $\sigma$ будем называть тотальной, а в случае $\Sigma=\varnothing-$ вырожденной. Вычисление схемы программ $\pi$ в семантике $\sigma$ на состоянии памяти $\xi_{0}$ протекает по следующему сценарию. Обход схемы $\pi$ начинается из ее входа с исходным состоянием памяти $\xi_{0}$. При прохождении через преобразователь с о.с. $s$ текущее состояние памяти $\xi_{t}$ изменяется на $\xi_{t+1}=I s\left(\xi_{t}\right)$, и обход $\pi$ продолжается по единственной дуге, исходящей из данного преобразователя. При прохождении через распознаватель с л. п. $p$ текущее состояние памяти $\xi_{t}$ не изменяется и обход схемы продолжается по дуге, помеченной символом $\operatorname{Ip}\left(\xi_{t}\right)$. Путь в схеме $\pi$, пройденный в процессе такого обхода называется вычислительной трассой. Если вычисление достигает выхода схемы, то оно считается успешным, а состояние памяти, образованное к этому моменту, -- результатом вычисления схемы на исходном состоянии $\xi_{0}$. Иначе полагаем, что вычисление схемы $\pi$ зацикливается и результат его неопределен. Нетрудно видеть, что в случае, когда трасса успешного вычисления схемы на исходном состоянии $\xi_{0}$ проходит последовательно через преобразователи, помеченные о.с. $s_{1}, s_{2}, \ldots, s_{n}$, образующими о. ц. $h=s_{1} s_{2} \ldots s_{n}$, результат вычисления будет равен $I h\left(\xi_{0}\right)$.

Схема $\pi$ в контексте заданной семантики $\sigma=\left\langle\Sigma, \Sigma_{0}, I\right\rangle$ определяет программу, вычисляющую частичную функцию $\Phi_{\pi, \sigma}: \Sigma_{0} \rightarrow \Sigma$. Для произвольного начального состояния памяти $\xi_{0}$ из $\Sigma_{0}$ значение $\Phi_{\pi, \sigma}\left(\xi_{0}\right)$ совпадает с результатом вычисления $\pi$, т.е. равно $\xi$, если вычисление схемы $\pi$ в семантике $\sigma$ на начальном состоянии памяти $\xi_{0}$ успешно завершается с результатом $\xi$, и неопределено, если вычисление $\pi$ на $\xi_{0}$ зацикливается. Две схемы $\pi^{\prime}$ и $\pi^{\prime \prime}$ считаются (функционально) эквивалентными в семантике $\sigma=\left\langle\Sigma, \Sigma_{0}, I\right\rangle$, если совпадают вычисляемые ими функции $\Phi_{\pi^{\prime}, \sigma}$ и $\Phi_{\pi^{\prime \prime}, \sigma}$. В вырожденной семантике все схемы считаются попарно эквивалентными.

Для абстрактных семантик над одним и тем же базисом $(S, P)$ введем операцию прямой суммы $\oplus$. Пусть $\sigma^{\prime}=\left\langle\Sigma^{\prime}, \Sigma_{0}^{\prime}, I^{\prime}\right\rangle, \sigma^{\prime \prime}=\left\langle\Sigma^{\prime \prime}, \Sigma_{0}^{\prime \prime}, I^{\prime \prime}\right\rangle$ и $\Sigma^{\prime} \cap \Sigma^{\prime \prime}=\varnothing$. Тогда прямое суммирование $\sigma^{\prime}$ и $\sigma^{\prime \prime}$ образует абстрактную семантику

$$
\sigma=\sigma^{\prime} \oplus \sigma^{\prime \prime}=\left\langle\Sigma^{\prime} \cup \Sigma^{\prime \prime}, \Sigma_{0}^{\prime} \cup \Sigma_{0}^{\prime \prime}, I\right\rangle,
$$

интерпретация $I$ которой определяется соотношениями

$$
\begin{aligned}
& I s(\xi)= \begin{cases}I^{\prime} s(\xi) & \text { при } \xi \in \Sigma^{\prime}, \\
I^{\prime \prime} s(\xi) & \text { при } \xi \in \Sigma^{\prime \prime},\end{cases} \\
& I p(\xi)= \begin{cases}I^{\prime} p(\xi) & \text { при } \xi \in \Sigma^{\prime}, \\
I^{\prime \prime} p(\xi) & \text { при } \xi \in \Sigma^{\prime \prime},\end{cases}
\end{aligned}
$$

для всех о.c. $s$ из $S$ и л. п. $p$ из $P$. Через $\sum_{\sigma \in \Omega} \sigma$ будем обозначать прямую сумму семантик множества $\Omega$. Если $\Sigma^{\prime} \cap \Sigma^{\prime \prime} \neq \varnothing$, то условимся обозначать через $\sigma^{\prime} \oplus$ $\sigma^{\prime \prime}$ прямую сумму изоморфных образов семантик $\sigma^{\prime}$ и $\sigma^{\prime \prime}$ с непересекающимися областями интерпретации.

Определим теперь основные понятия семантик иного типа - формальных моделей программ. Ознячиванием логических переменных символьного базиса будем 
называть всюду определенное отображение Val: $P \rightarrow\{0,1\}$. Обозначим через $B_{P}$ множество всевозможных означиваний л. п. базиса. Функцией разметки над базисом $(S, P)$ называется любая всюду определенная функция $\mu: S^{*} \rightarrow B_{P}$, сопоставляющая каждой о.ц. $h$ некоторое означивание $\mu h$ л. п. данного базиса. Множество всех функций разметки над базисом $(S, P)$ обозначим $\mathscr{L}(\mathscr{S}, \mathscr{P})$. Будем говорить, что функции $\mu^{\prime}$ и $\mu^{\prime \prime}$ имеют одинаковое поведение на о. ц. $H$, и обозначать $\mu^{\prime} \stackrel{H}{=} \mu^{\prime \prime}$, если для каждого префикса $h$ цепочки $H$ функции разметки $\mu^{\prime}$ и $\mu^{\prime \prime}$ задают одинаковые означивания л. п. $\mu^{\prime} h=\mu^{\prime \prime} h$.

Вычисление схемы $\pi$ на функции разметки $\mu$ осуществляется следующим образом. Обход схемы начинается из ее входа с пустой о.ц. е. Прохождение через преобразователь с о.ц. $s \in S$ сопровождается приписыванием $s$ к текущей о.ц. $h_{t}$. При прохождении через преобразователь с л. п. $p$ по функции разметки $\mu$ и текущей о. ц. $h_{t}$ определяется означивание $\mu h_{t}$ и значение $\mu h_{t}(p)=\delta$ л. п. $p$, после чего обход продолжается по дуге с пометкой $\delta$, исходящей из данного распознавателя, без изменения о. ц. $h_{t}$. Процедура вычисления завершается по достижении выхода схемы. Построенная к этому моменту о.ц. считается результатом вычисления схемы $\pi$ на функции разметки $\mu$, а пройденный путь в графе $\pi$ - трассой вычисления. Если выход схемы недостижим, то результат вычисления считается неопределенным. Под этапом (или тактом) вычисления будем понимать отрезок вычислительной трассы между двумя последовательно пройденными преобразователями.

Описанная процедура вычисления позволяет сопоставить каждой схеме $\pi$ над базисом $(S, P)$ частичный функционал $F_{\pi}: \mathscr{L}(\mathscr{S}, \mathscr{P}) \rightarrow \mathscr{S}^{*}$. Значение $F_{\pi}(\mu)$ определено и равно $h$ тогда и только тогда, когда вычисление схемы $\pi$ на функции разметки $\mu$ завершается с результатом $h$. Вполне очевидно следующее утверждение.

Предложение 1. Если $\mu^{\prime} \stackrel{h}{=} \mu^{\prime \prime}$ и при вычислении схемы $\pi$ на функчии разметки $\mu^{\prime}$ будет построена о. и. $h$, то вычисление $\pi$ на бункции разметки $\mu^{\prime \prime}$ приводит $\kappa$ построению той же самой о. u. $h$.

Следствие 1. Если $\mu^{\prime} \stackrel{h}{=} \mu^{\prime \prime} u F_{\pi}\left(\mu^{\prime}\right)=h$, mo $F_{\pi}\left(\mu^{\prime \prime}\right)=h$.

Формальная модель программ над символьным базисом $(S, P)$ задается двумя параметрами: отношением эквивалентности $\tau$ на $S^{*}$ и множеством функций разметки $L \subseteq \mathscr{L}(\mathscr{S}, \mathscr{P})$. Формальную модель такого вида будем обозначать $K(\tau, L)$. Схемы $\pi^{\prime}$ и $\pi^{\prime \prime}$ считаются эквивалентными в модели $K(\tau, L)$, или, иначе говоря, $(\tau, L)$-эквивалентными $\left(\pi^{\prime} \simeq_{\tau, L} \pi^{\prime \prime}\right)$, если для любой функции разметки $\mu$ из $L$ либо оба значения $F_{\pi^{\prime}}(\mu)$ и $F_{\pi^{\prime \prime}}(\mu)$ не определены, либо $F_{\pi^{\prime}}(\mu)=h^{\prime}, F_{\pi^{\prime \prime}}(\mu)=h^{\prime \prime}$ и $h^{\prime} \tau h^{\prime \prime}$ (т. е. $h^{\prime}$ и $h^{\prime \prime}-\tau$-эквивалентные о. ц.).

Простым замыканием множества $L \subseteq \mathscr{L}(\mathscr{S}, \mathscr{P})$ называется множество функций разметки

$$
[L]=\left\{\mu: \forall h \in S^{*} \exists \mu^{\prime} \in L\left(\mu^{\prime} \stackrel{h}{=} \mu^{\prime \prime}\right)\right\} .
$$

Если $L=[L]$, то множество функций разметки $L$ и формальная модель $K(\tau, L)$ называются гладкими. Функция разметки $\mu \in \mathscr{L}(\mathscr{S}, \mathscr{P})$ называется согласованной с отношением эквивалентности $\tau$ на $S^{*}$ (или $\tau$-согласованной), если для любой пары $\tau$-эквивалентных о.ц. $h^{\prime}$ и $h^{\prime \prime}$ означивания $\mu h^{\prime}$ и $\mu h^{\prime \prime}$ совпадают. Условимся обозначать через $L_{\tau}$ подмножество $\tau$-согласованных функций разметки из множества $L$. 
Если $L=L_{\tau}\left(L=[L]_{\tau}\right)$, то формальную модель $K(\tau, L)$ будем называть согласованной (согласованно-гладкой). Согласованную формальную модель программ $K(\tau, L)$ назовем правоконгруэнтной моделью, если отношение эквивалентности $\tau$ является правой конгруэнцией на множестве о.ц. $S^{*}$ (т. е. для любых о. ц. $h_{1}, h_{2}, h_{3}$ из $h_{1} \tau h_{2}$ следует $\left.h_{1} h_{3} \tau h_{2} h_{3}\right)$. Для произвольного о.с. $s$ из $S$ и функции разметки $\mu$ из $\mathscr{L}(\mathscr{S}, \mathscr{P})$ назовем сдвигом $\mu_{s}$ функцию разметки, значение которой на всякой о. ц. $h$ определяется соотношением $\mu_{s} h=\mu s h$. Согласованную формальную модель $K(\tau, L)$ будем называть полугрупповой моделью, если отношение эквивалентности $\tau$ является конгруэнцией на $S^{*}$ (т. е. для любых о.ц. $h_{1}, h_{2}, h_{3}, h_{4}$ из $h_{1} \tau h_{2}$ и $h_{3} \tau \cdot h_{4}$ следует $h_{1} h_{3} \tau h_{2} h_{4}$ ), а множество $L$ замкнуто относительно операторного сдвига (т.е. для любой функции разметки $\mu$ из $L$ и о.с. $s$ из $S$ сдвиг $\mu_{s}$ также принадлежит $L)$.

На множестве формальных моделей над общим базисом $(S, P)$ можно ввести отношение сравнимости. Модель $K\left(\tau^{\prime}, L^{\prime}\right)$ будем считать не менее выразительной, чем $K\left(\tau^{\prime \prime}, L^{\prime \prime}\right)$ и обозначать $K\left(\tau^{\prime}, L^{\prime}\right) \leqslant K\left(\tau^{\prime \prime}, L^{\prime \prime}\right)$, если для любой пары схем $\pi_{1}, \pi_{2}$ эквивалентность $\pi_{1} \simeq_{\tau, L^{\prime}} \pi_{2}$ влечет $\pi_{1} \simeq_{\tau, L^{\prime \prime}} \pi_{2}$. Формальные модели $K\left(\tau^{\prime}, L^{\prime}\right)$ и $K\left(\tau^{\prime \prime}, L^{\prime \prime}\right)$ считаются равносильными $\left(K\left(\tau^{\prime}, L^{\prime}\right) \approx K\left(\tau^{\prime \prime}, L^{\prime \prime}\right)\right)$, если $K\left(\tau^{\prime}, L^{\prime}\right) \leqslant$ $K\left(\tau^{\prime \prime}, L^{\prime \prime}\right)$ и $K\left(\tau^{\prime \prime}, L^{\prime \prime}\right) \leqslant K\left(\tau^{\prime}, L^{\prime}\right)$. Подобным же образом можно провести сравнение абстрактных семантик, а также разнотипных семантик над одним и тем же базисом. Будем говорить, что формальная модель программ $K(\tau, L)$ аппроксимирует абстрактную семантику $\sigma$, если для всякой пары схем $\pi_{1}$ и $\pi_{2}$ эквивалентность $\pi_{1} \simeq_{\tau, L} \pi_{2}$ влечет $\pi_{1} \simeq_{\sigma} \pi_{2} ; K(\tau, L)$ будем при этом называть аппроксимацией, или приближением, семантики $\sigma$. Основной исследовательский вопрос настоящей статьи таков.

(1) При каких условиях формальная модель программ $K(\tau, L)$ аппроксимирует заданную абстрактную семантику $\sigma$ ?

При моделировании одних функциональных систем другими значительный интерес вызывают наиболее точные модели. Аппроксимацию $K(\tau, L)$ семантики $\sigma$ назовем минимальной, если для любой формальной модели $K\left(\tau^{\prime}, L^{\prime}\right)$ из $\sigma \leqslant K\left(\tau^{\prime}, L^{\prime}\right) \leqslant$ $K(\tau, L)$ следует $K\left(\tau^{\prime}, L^{\prime}\right) \approx K(\tau, L)$. Аппроксимация $K(\tau, L)$ семантики $\sigma$ будет считаться наилучшей, если для всякой другой аппроксимации $K\left(\tau^{\prime}, L^{\prime}\right)$ семантики $\sigma$ выполняется $K(\tau, L) \leqslant K\left(\tau^{\prime}, L^{\prime}\right)$. В тех случаях, когда наше внимание ограничено определенным классом формальных моделей, будем говорить о минимальной или наилучшей аппроксимации заданной семантики $\sigma$ в рассматриваемом классе моделей. В связи с этим уместен следующий вопрос :

(2) При каких условиях абстрактная семантика $\sigma$ имеет наилучшую (минимальную) аппроксимацию в тех или иных классах моделей?

Среди формальных моделей, наиболее точно приближающих абстрактные семантики, особое место занимают модели, равносильные некоторым абстрактым семантикам. Поэтому изучению подлежит также и вопрос :

(3) При каких условиях формальная модель $K(\tau, L)$ имеет равносильную абстрактную семантику? 


\section{3. Предшествующие результаты}

Решение поставленных задач имеет как прикладное, так и чисто математическое значение. С позиции современного программирования нетрудно проследить взаимосвязь абстрактных семантик программ и операционных семантик систем процедурного программирования, базирующихся на языке логики предикатов первого порядка. Достоинства формальных моделей программ состоят в том, что для определения параметров $\tau$ и $L$ и описания процедуры вычисления схемы программ могут быть привлечены алгебраические, теоретико-автоматные и формально-языковые конструкции. Благодаря этому обстоятельству проблемы эквивалентности, включения и пустоты схем программ в некоторых формальных моделях сводятся к соответствующим проблемам в указанных теориях. Так, например, проблема эквивалентности схем программ в так называемых автоматных формальных моделях вида $K\left(\varepsilon, L_{r}\right)[11,12]$, обобщающих схемы Янова $[13,14]$ (здесь $\varepsilon$ - тождественное отношение эквивалентности о. ц., а множество функций разметки $L_{r}$ задается регулярным образом), сводится к проблеме эквивалентности конечных автоматов. В $[8,15-18]$ была исследована проблема эквивалентности схем программ в так называемых транзитивно-коммутативных формальных моделях $K\left(\tau, L_{\tau}\right)$, отношение эквивалентности $\tau$ в которых задается разбиением множества о. с. $S$ на непересекающиеся подмножества перестановочных символов. При наложении эквивалентности $\tau$ на $S^{*}$ полугруппа о. ц. будет представима в виде свободного произведения свободных коммутативных полугрупп, порожденных указанными подмножествами перестановочных о.с. Было установлено, что при некоторых множествах разметки $L$ проблема эквивалентности схем программ в транзитивно-коммутативных моделях сводится либо к разрешимой проблеме эквивалентности конечных автоматов над свободной коммутативной полугруппой [19], либо к разрешимому частному случаю проблемы эквивалентности многоленточных автоматов с непересекающимися циклами [20], либо к общей проблеме эквивалентности конечных многоленточных автоматов. Широкий спектр разрешимых и неразрешимых случаев проблемы эквивалентности дискретных преобразователей Глушкова-Летичевского, соответствующих полугрупповым моделям программ вида $K\left(\tau, \mathscr{L}(\mathscr{S}, \mathscr{P})_{\tau}\right)$ рассмотрен в [21]. Таким образом, формальные модели программ $K(\tau, L)$, аппроксимирующие абстрактную семантику $\sigma$, играют роль промежуточного канала, в котором процедуры, разрешающие некоторые алгоритмические проблемы алгебры, теории автоматов и формальных языков, адаптируются для получения частичного решения задач анализа вычислительных программ в семантике $\sigma$.

Вопросы приближения тотальных абстрактных семантик формальными моделями программ в несколько иной постановке изучались в работах $[3,4,6]$. Будем говорить, что формальная модель $K(\tau, L)$ приближает некоторое множество абстрактных семантик $\Omega$, если $\sigma \leqslant K(\tau, L)$ для любой семантики $\sigma$ из $\Omega$. Формальная модель $K(\tau, L)$ считается равносильной множеству абстрактных семантик $\Omega$, если для любой пары схем программ $\pi^{\prime}$ и $\pi^{\prime \prime}$ справедливо соотношение

$$
\pi^{\prime} \simeq_{\tau, L} \pi^{\prime \prime} \Longleftrightarrow \forall \sigma \in \Omega\left(\pi^{\prime} \simeq_{\sigma} \pi^{\prime \prime}\right) .
$$

Равносильность модели $K(\tau, L)$ множеству семантик $\Omega$ означает, что данная формальная модель полностью отражает все общие свойства абстрактных семантик семейства $\Omega$. В [3] было установлено достаточное условие аппроксимируемости множества тотальных семантик $\Omega$ формальной моделью $K(\tau, L)$. Было выяснено 
также, что для одного семейства семантик - тотальных семантик с гладким моноядром - данное условие является критерием аппроксимируемости. В [4] было показано, что для каждой полугрупповой модели $K(\tau, L)$ имеется класс абстрактных семантик $\Omega$, равносильный $K(\tau, L)$, и при этом максимальная формальная модель $K(\varepsilon, \mathscr{L}(\mathscr{S}, \mathscr{P}))$ равносильна семейству всевозможных абстрактных семантик над базисом $(S, P)$. В [6] было обнаружено, что для каждой структуризации символьного базиса $(S, P)$ семейство всевозможных тотальных семантик структурированных схем программ над базисом $(S, P)$ имеет равносильную полугрупповую формальную модель. Последнее означает, что одна из основных задач теории схем программ - проблема функциональной эквивалентности структурированных схем программ $[9,22]$ - может быть выражена в терминах эквивалентности схем в формальных моделях программ специального вида. Как следует из приведенного ниже утверждения, проблемы аппроксимации семейств абстрактных семантик формальными моделями могут быть сведены к соответствующим задачам аппроксимации индивидуальных абстрактных семантик.

Предложение 2. Для семантик $\sigma_{1} u \sigma_{2}$

$$
\left(\sigma_{1} \leqslant K(\tau, L)\right) \&\left(\sigma_{2} \leqslant K(\tau, L)\right) \Longleftrightarrow \sigma_{1} \oplus \sigma_{2} \leqslant K(\tau, L) .
$$

Следствие 2. Если $\Omega$ - некоторое множество абстрактных семантик над символьным базисом $(S, P)$, по формалъная модель $K(\tau, L)$ аппроксимирует $\Omega$ (равносилъна $\Omega$ ) тогда и толъко тогда, когда

$$
\sum_{\sigma \in \Omega} \sigma \leqslant K(\tau, L) \quad\left(\sum_{\sigma \in \Omega} \sigma \approx K(\tau, L)\right) .
$$

При определении условий апроксимируемости абстрактных семантик формальными моделями программ большую роль играет отношение сравнимости формальных моделей. Анализ свойств этого отношения был проведен в работах $[3,23,24]$. Было установлено необхоцимое

$$
K\left(\tau^{\prime}, L^{\prime}\right) \leqslant K\left(\tau^{\prime \prime}, L^{\prime \prime}\right) \Rightarrow\left(\tau^{\prime \prime} \rightarrow \tau^{\prime}\right) \&\left(\left[L^{\prime}\right] \subseteq\left[L^{\prime \prime}\right]\right)
$$

и достаточное

$$
\left(\tau^{\prime \prime} \rightarrow \tau^{\prime}\right) \&\left(L^{\prime} \subseteq L^{\prime \prime}\right) \Rightarrow K\left(\tau^{\prime}, L^{\prime}\right) \leqslant K\left(\tau^{\prime \prime}, L^{\prime \prime}\right)
$$

условия сравнимости формальных моделей и показано, что для некоторых классов формальных моделей (гладких, согласованно-гладких и др.) соотношения $\tau^{\prime \prime} \rightarrow \tau^{\prime}$ и $L^{\prime} \subseteq L^{\prime \prime}$ являются критерием сравнимости моделей $K\left(\tau^{\prime}, L^{\prime}\right)$ и $K\left(\tau^{\prime \prime}, L^{\prime \prime}\right)$. В то же время имеются сравнимые по отношению $\leqslant$ модели $K\left(\tau_{1}, L_{1}\right), K\left(\tau_{2}, L_{2}\right)$ такие, что $K\left(\tau_{1}, L_{1}\right) \leqslant K\left(\tau_{2}, L_{2}\right)$, но при этом $L_{2} \subset L_{1}[23]$. В свете соотношений.(1), (2) ясно также значение наилучшей формальной аппроксимации абстрактной семантики: если $\sigma$ имеет наилучшее формальное приближение $K(\tau, L)$, то указанные соотношения предоставляют необходимое и достаточное условие аппроксимируемости $\sigma$ формальными моделями программ.

Отношение $\leqslant$ сравнимости формальных моделей программ является отношением квазипорядка и становится отношением частичного порядка, будучи распространенным естественным образом на фактор-множество формальных моделей по отношению равносильности. Немаловажную роль при решении задач аппроксимации играют решетчатые свойства данного фактор-множества. 
Предложение 3. Если $\sigma^{\prime}=\left\langle\Sigma, \Sigma^{\prime}, I\right\rangle, \sigma^{\prime \prime}=\left\langle\Sigma, \Sigma^{\prime \prime}, I\right\rangle$, mо $\sigma^{\prime} \oplus \sigma^{\prime \prime} \approx\left\langle\Sigma, \Sigma^{\prime} \cup \Sigma^{\prime \prime}, I\right\rangle$.

Предложение 4. Если абстрактные семантики $\sigma^{\prime}$ и $\sigma^{\prime \prime}$ имеют наилучшие формальные аппроксимации $K\left(\tau^{\prime}, L^{\prime}\right)$ u $K\left(\tau^{\prime \prime}, L^{\prime \prime}\right)$, то формальная модель $K(\tau, L)$ будет служить приближением семантики $\sigma=\sigma^{\prime} \oplus \sigma^{\prime \prime}$ тогда и только тогда, когда $K\left(\tau^{\prime}, L^{\prime}\right) \leqslant K(\tau, L) u K\left(\tau^{\prime \prime}, L^{\prime \prime}\right) \leqslant K(\tau, L)$.

Следствие 3. Есои абстрактные семантики $\sigma^{\prime}$ u $\sigma^{\prime \prime}$ имеют наилучшие аппроксимачии $K\left(\tau^{\prime}, L^{\prime}\right)$ и $K\left(\tau^{\prime \prime}, L^{\prime \prime}\right)$ на некотором множестве формальных моделей $\mathscr{K}$, то семантика $\sigma=\sigma^{\prime} \oplus \sigma^{\prime \prime}$ имеет наилучшее приближение в $\mathscr{K}$ тогда и только тогда, когда классы равносильности $K(\tau, L) / \approx u K(\tau, L) / \approx$ имеют точную верхнюю грань в фактор-множестве $\mathscr{K} / \approx$.

Теорема 1. Пусть $\sigma=\left\langle\Sigma, \Sigma_{0}, I\right\rangle$, и фактор-множество $\mathscr{K} / \approx$ некоторого семейства формалъных моделей $\mathscr{K}$ образует полную верхнюю полурешетку по отношению сравнимости $\leqslant$. Тогда $\sigma$ имеет наилучшую формальную аппроксимацию в $\mathscr{K}$, если для всякого $\xi_{0}$ из $\Sigma_{0}$ абстрактная семантика $\left\langle\Sigma,\left\{\xi_{0}\right\}, I\right\rangle$ имеет наилучшее приближение в $\mathscr{K}$.

Доказательство. Исходя из определения прямой суммы абстрактных семантик и соглашения о суммировании семантик с общей областью интерпретации нетрудно заметить, что для любого множества начальных состояний $\Sigma_{0} \subseteq \Sigma$ выполняется соотношение

$$
\left\langle\Sigma, \Sigma_{0}, I\right\rangle \approx \sum_{\xi \in \Sigma_{0}}\langle\Sigma,\{\xi\}, I\rangle
$$

Тогда справедливость теоремы следует из предшествующего ей предложения 4.

В $[3,23]$ было установлено, что фактор-множества гладких, согласованно-гладких моделей, а также всевозможных моделей $K(\tau, L)$ с произвольным фиксированным отношением эквивалентности о. ц. $\tau$ образуют полную решетку по отношению сравнимости $\leqslant$. Вопрос о решетчатых свойствах фактор-множества всех формальных моделей над общим базисом оставался открытым. Как будет установлено в настоящей работе, это частично-упорядоченное множество не является верхней полурешеткой.

\section{4. Правоконгруэнтные формальные модели и частичные абстрактные семантики программ}

Теорема 2. Каждая правоконгруэнтная модель программ $K(\tau, L)$ имеет равносильную частичную абстрактную семантику $\sigma$.

Доказательство. Рассмотрим произвольную правоконгруэнтную модель $K(\tau, L)$. Для каждой о. ц. $h$ обозначим через $[h]$ класс $\tau$-эквивалентности на $S^{*}$, содержащий $h$. Формальной модели $K(\tau, L)$ сопоставим абстрактную семантику $\sigma=\left\langle\Sigma, \Sigma_{0}, I\right\rangle$ следующего вида :

(1) $\Sigma=\left\{\xi_{\mu[h]}: \mu \in L,[h] \in S^{*} / \tau\right\}$, т. е. каждое состояние памяти из $\Sigma$ взаимнооднозначно соответствует паре $(\mu,[h])$;

(2) $\Sigma_{0}=\left\{\xi_{\mu[e]}: \mu \in L, e-\right.$ пустая о.ц. $\}$; 
(3) для каждого $s \in S$ и $\xi_{\mu[h]} \in \Sigma$ значение $I s\left(\xi_{\mu[h]}\right)=\xi_{\mu[h s]}$;

(4) для каждого $p \in P$ и $\xi_{\mu[h]} \in \Sigma$ значение $I p\left(\xi_{\mu[h]}\right)=\mu h(p)$.

Корректность определения $\sigma$ следует из свойств правой конгруэнции $\left(h_{1} \in[h]\right.$, $h_{2} \in[h]$ влечет $h_{1} s \in[h s]$ и $\left.h_{2} s \in[h s]\right)$ и $\tau$-согласованности функций разметки $\left(h_{1} \tau h_{2}\right.$ влечет совпадение означиваний $\left.\mu h_{1}=\mu h_{2}\right)$. Исходя из данного определения $\sigma$ индукцией по длине $t$ вычислительной трассы можно показать, что для любой схемы $\pi$ вычисление в семантике $\sigma$ на начальном состоянии памяти $\xi_{\mu[e]}$ достигает на $t$-м этапе вершины $\nu$ с результатом $\xi_{\mu[h]}$ тогда и только тогда, когда вычисление $\pi$ в модели $K(\tau, L)$ на функции разметки $\mu$ достигает на $t$-м этапе той же вершины $\nu$ с результатом $h^{\prime} \in[h]$. Поэтому для любых схем $\pi_{1}$ и $\pi_{2}$

$$
\pi_{1} \simeq_{\sigma} \pi_{2} \Longleftrightarrow \pi_{1} \simeq_{\tau, L} \pi_{2}
$$

Построенную при доказательстве теоремы 2 семантику $\sigma$, равносильную правоконгруэнтной модели $K(\tau, L)$, будем в дальнейшем обозначать $\sigma(\tau, L)$.

Как будет показано далее, обратное утверждение о том, что всякая абстрактная семантика имеет равносильную формальную модель, в общем случае неверно, поскольку выразительные возможности абстрактных программных семантик существенно выше, нежели выразительные средства аппарата формальных моделей. По этой причине в теории формальных моделей программ вместо задачи построения для заданной абстрактной семантики $\sigma$ равносильной формальной модели приходится исследовать более общую задачу поиска наилучшего приближения семантики $\sigma$ в том или ином классе формальных моделей. На первых подступах к ее разрешению рассмотрим один простой метод построения специального формального приближения для произвольной семантики $\sigma=\left\langle\Sigma, \Sigma_{0}, I\right\rangle$. Обозначим через $K(\tau(\sigma), L(\sigma))$ формальную модель, параметры которой удовлетворяют следующим требованиям :

для любых о.ц. $h_{1}, h_{2}$ эквивалентность $h_{1} \tau(\sigma) h_{2}$ имеет место тогда и только тогда, когда для каждого начального состояния памяти $\xi$ из множества $\Sigma_{0}$ выполняется $I h_{1}(\xi)=I h_{2}(\xi)$;

$L(\sigma)=\left\{\mu_{\xi}: \xi \in \Sigma_{0}\right\}$, где через $\mu_{\xi}$ обозначены функции разметки, которые на каждой о. ц. $h$ задают означивания $\mu_{\xi} h$, подчиняющиеся соотношению $\mu_{\xi} h(p)=$ $\operatorname{Ip}(\operatorname{Ih}(\xi))$ для всякой л. п. $p$ из $P$.

Как видно из приведенного определения, трасса вычисления произвольной схемы $\pi$ на начальном состоянии памяти $\xi$ из $\Sigma_{0}$ в семантике $\sigma$ совпадает с трассой вычисления $\pi$ на функции разметки $\mu_{\xi}$. Принимая во внимание требования, наложенные на $\tau(\sigma)$, можно заключить, что формальная модель $K(\tau(\sigma), L(\sigma))$ является аппроксимацией семантики $\sigma$. Эту модель будем называть стандартной аппроксимацией $\sigma$. Отметим следующие основные свойства стандартной аппроксимации.

Предложение 5. $K(\tau(\sigma), L(\sigma))$ является правоконгруэнтной моделъю.

Предложение 6. Для любой аппроксимации $K(\tau, L)$ семантики $\sigma$ выполняются соотношения

$$
\begin{gathered}
\tau \rightarrow \tau(\sigma), \\
{[L(\sigma)] \subseteq[L] .}
\end{gathered}
$$



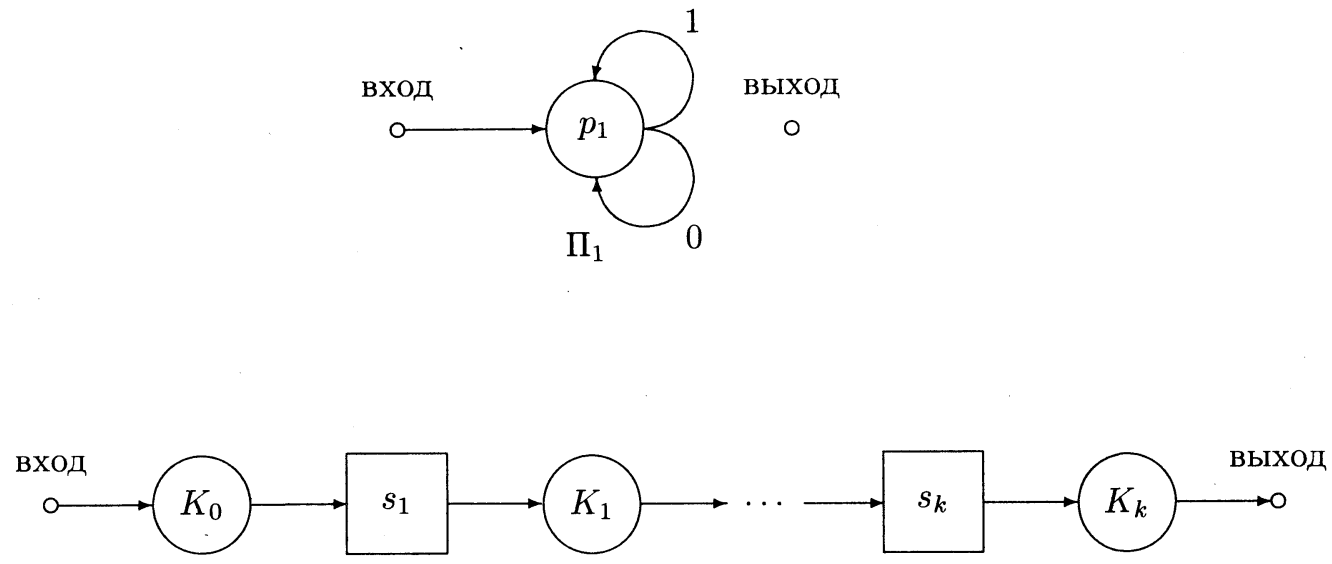

$\Pi_{2}$

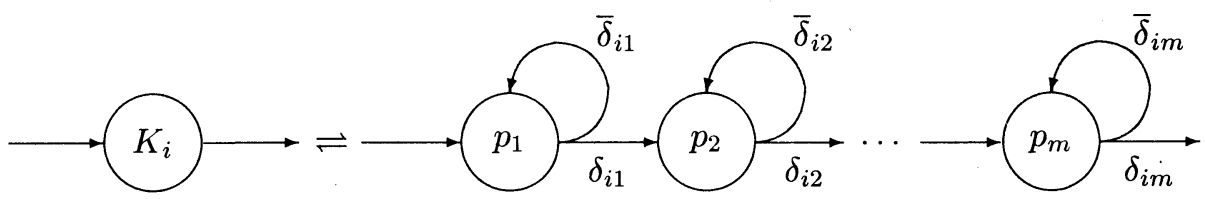

Pис. 1.

Доказательство. В случае невыполнимости первого соотношения для некоторой пары $\tau$-эквивалентных о. ц. $h_{1}$ и $h_{2}$ имелось бы начальное состояние памяти $\zeta$ такое, что $I h_{1}(\zeta) \neq I h_{2}(\zeta)$. Рассмотрим схемы $\pi_{1}$ и $\pi_{2}$, каждая из которых представляет собой цепочку преобразователей, соединяющую вход и выход, причем преобразователям схемы $\pi_{i}, i=1,2$, последовательно приписаны о.с., составляющие о. . $h_{i}, i=1,2$. Данные схемы эквивалентны в модели $K(\tau, L)$, но неэквивалентны в семантике $\sigma$ вопреки предположению о том, что $K(\tau, L)$ аппроксимирует $\sigma$.

Согласно определению операции простого замыкания множества функций разметки, невыполнимость второго соотношения означает, что $L(\sigma)$ содержит функцию разметки $\mu_{\xi}$, поведение которой на некоторой о. ц. $h=s_{1} s_{2} \ldots s_{k}$ отлично от поведения любой функции разметки из множества $L$. Тогда схемы $\Pi_{1}$ и $\Pi_{2}$, изображенные на рис. 1 (через $K_{i}, 0 \leqslant i \leqslant k$, обозначены логические фрагменты, соответствующие элементарным конъюнкциям для множества $\left\{\mu_{\xi} s_{1} \ldots s_{i}(p): p \in P\right\}$ значений всех л. п.), эквивалентны в модели $K(\tau, L)$, так как на множестве функций разметки $L$ ими вычисляются всюду неопределенные функционалы $F_{\Pi_{1}}$ и $F_{\Pi_{2}}$, но неэквивалентны в семантике $\sigma$ ибо для начального состояния памяти $\xi$ значение $\Phi_{\Pi_{1}, \sigma}(\xi)$ неопределено, а $\Phi_{\Pi_{2}, \sigma}(\xi)=I h(\xi)$. Возникающее противоречие с предположением об аппроксимируемости $\sigma$ моделью $K(\tau, L)$ устраняется при $[L(\sigma)] \subseteq[L]$.

Предложение 7. Если $K(\tau, L)$ - правоконгруэнтная моделъ $u K\left(\tau, L^{\prime}\right) \leqslant K(\tau, L)$, то $K\left(\tau, L^{\prime}\right)$ также является правоконгруэнтной моделъю.

Доказательство. Достаточно установить, что каждая функция разметки из множества $L^{\prime}$ согласована с правой конгруэнцией $\tau$. Допустим, что $L^{\prime}$ содержит функ- 

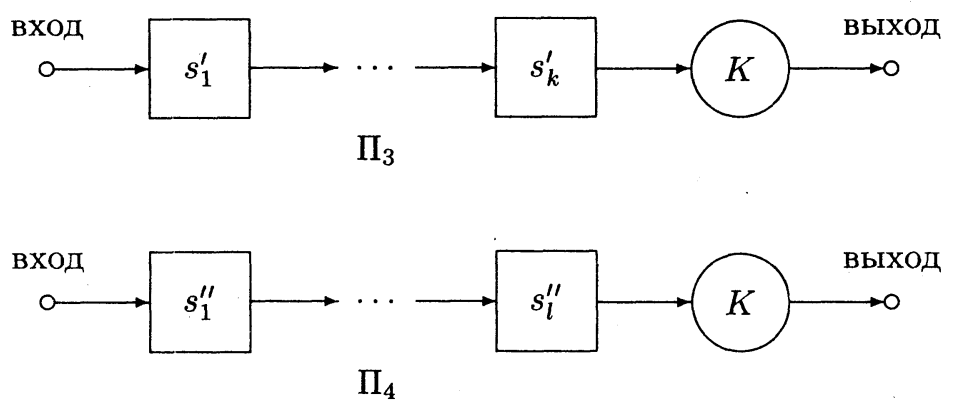

Pис. 2.

цию разметки $\mu$ такую, что для некоторой пары $\tau$-эквивалентных о.ц. $h$ и $H$ выполняется $\mu h \neq \mu H$. Рассмотрим схемы программ $\Pi_{3}$ и $\Pi_{4}$, изображенные на рис. 2 . Здесь, как и на рис. 1 , используется логический фрагмент $K$, соответствующий элементарной конъюнкции $p_{1}^{\delta_{1}} \& \ldots \& p_{m}^{\delta_{m}}$ по всем л. п. базиса, где $\delta_{i}=\mu H\left(p_{i}\right)$, $i=1, \ldots, m$. Поскольку множество $L$ состоит из $\tau$-согласованных функций разметки, схемы $\Pi_{3}$ и $\Pi_{4}$ эквивалентны в модели $K(\tau, L)$. В то же времы на функции разметки $\mu$ из $L^{\prime}$ значение $F_{\Pi_{3}}(\mu)$ не определено, а $F_{\Pi_{4}}(\mu)=H$. Поэтому $\Pi_{3} \simeq_{\tau, L} \Pi_{4}$ вопреки тому, что $K\left(\tau, L^{\prime}\right) \leqslant K(\tau, L)$. Полученное противоречие означает, что все функции разметки множества $L^{\prime}$ должны быть $\tau$-согласованными, т. е. $K\left(\tau, L^{\prime}\right)$ является правоконгруэнтной моделью.

Из предложений 5-7 можно вывести следующую теорему.

Теорема 3. Если абстрактная семантика $\sigma$ имеет наилучшую аппроксимацию $K(\tau, L)$, то $K(\tau, L)-$ правоконгруэнтная модель, $\tau=\tau(\sigma) u L \subseteq[L(\sigma)]_{\tau(\sigma)}$.

Доказательство. Обратимся к стандартной аппроксимации $K(\tau(\sigma), L(\sigma))$ семантики $\sigma$. Поскольку $K(\tau, L)$ - наилучшая аппроксимация $\sigma$, справедливо отношение $K(\tau, L) \leqslant K(\tau(\sigma), L(\sigma))$. Из предложения 6 и необходимого условия (1) сравнимости формальных моделей следует равенство $\tau=\tau(\sigma)$ и включение $L \subseteq[L]=[L(\sigma)]$. Из предложений 5 и 7 следует, что формальная модель $K(\tau, L)$ является правоконгруэнтной. Поскольку множество $L$ состоит из $\tau$-согласованных функций разметки, справедливо включение $L \subseteq[L(\sigma)]_{\tau(\sigma)}$.

Следствие 4. Если абстрактная семантика б равносильна формальной модели $K(\tau, L)$, то $K(\tau, L)$ - правоконгруэнтная моделъ.

Как будет установлено в пятом разделе, существуют абстрактные семантики $\sigma$, для которых стандартная аппроксимация $K(\tau(\sigma), L(\sigma))$ не является не только наилучшим, но даже минимальным приближением. Тем не менее, для некоторых типов семантик $\sigma$ модель $K(\tau(\sigma), L(\sigma))$ будет наилучшей аппроксимацией в классе согласованных формальных моделей. Простейшим примером тому могут служить абстрактные семантики, область начальных данных которых состоит из единственного состояния памяти. 
Предложение 8. Если $\sigma=\left\langle\Sigma, \Sigma_{0}, I\right\rangle$ u $\Sigma_{0}=\left\{\xi_{0}\right\}$, mo $\sigma \approx K(\tau(\sigma), L(\sigma))$.

Доказательство утверждения вытекает непосредственно из определения стандартной аппроксимации абстрактной семантики $\sigma$.

Теорема 4. Всякая абстрактная семантика $\sigma$ имеет наилучшее формалъное приближение в классах гладких и согласованно-гладких моделей.

Доказательство. Согласно предложению 8 любая семантика $\sigma_{\xi}=\langle\Sigma,\{\xi\}, I\rangle$ имеет наилучшее формальное приближение $K\left(\tau\left(\sigma_{\xi}\right), L\left(\sigma_{\xi}\right)\right)$, где $L\left(\sigma_{\xi}\right)=\left\{\mu_{\xi}\right\}$. Поскольку $\mu_{\xi}-\tau\left(\sigma_{\xi}\right)$-согласованная функция разметки и $\left[\left\{\mu_{\xi}\right\}\right]=\left\{\mu_{\xi}\right\}$, формальная модель $K\left(\tau\left(\sigma_{\xi}\right), L\left(\sigma_{\xi}\right)\right)$ является гладкой и согласованной. Как было установлено в [23], фактор-множества классов гладких и согласованно-гладких моделей образуют полную решетку по отношению сравнимости $\leqslant$. Тогда утверждение данной теоремы следует из теоремы 1 . Ввиду того, что соотношения $\tau_{2} \rightarrow \tau_{1}$ и $L_{1} \subseteq L_{2}$ служат критерием сравнимости гладких и согласованно-гладких моделей $[3,23]$, можно показать, исходя из предложения 6 и теоремы 3 , что $K(\tau(\sigma),[L(\sigma)])$ будет наилучшим приближением абстрактной семантики $\sigma$ в классе гладких моделей, а $K\left(\tau(\sigma),[L(\sigma)]_{\tau(\sigma)}\right)$ в классе согласованно-гладких моделей.

Бо ле изощренным примером абстрактных семантик, имеющих наилучшие формальные приближения, являются обратимые семантики. Абстрактную семантику $\sigma=\left\langle\Sigma, \Sigma_{0}, I\right\rangle$ над символьным базисом $(S, P)$ назовем обратимой, если для любого о. c. $s$ существует о. ц. $h$ такая, что равенство $\operatorname{Ih}(\operatorname{Is}(\xi))=I s h(\xi)=\xi$ выполняется для всякого состояния памяти $\xi$ из $\Sigma$. Указанную о.ц. $h$, соответствующую о.с. $s$, будем обозначать $s^{-1}$. Обратимость семантики $\sigma$ является необходимым и достаточным условием того, что преобразования памяти $I h$, порождаемые о. ц. из $S^{*}$, образуют группу отображений на множестве $\Sigma$ относительно операции композиции.

Теорема 5. Если $\sigma=\left\langle\Sigma, \Sigma_{0}, I\right\rangle$ - обратимая семантика, то стандартная аппроксимачия $K(\tau(\sigma), L(\sigma))$ является минимальным приближением $\sigma$ в классе всевозможных формальных моделей.

Доказательство. Рассмотрим произвольную формальную модель $K(\tau, L)$, удовлетворяюшую отношению $\sigma \leqslant K(\tau, L) \leqslant K(\tau(\sigma), L(\sigma))$, и покажем, что стандартная аппроксимация $K(\tau(\sigma), L(\sigma))$ равносильна $K(\tau, L)$. Прежде всего отметим, что в силу предложений 6,7 и необходимого условия (1) сравнимости формальных моделей отношение эквивалентности $\tau$ совпадает о $\tau(\sigma),[L]=[L(\sigma)]$, и множество $L$ состоит из $\tau(\sigma)$-согласованных функций разметки.

Допустим, что некоторые произвольные схемы $\pi^{\prime}$ и $\pi^{\prime \prime}$ эквивалентны в модели $K(\tau, L)$. Из $\sigma \leqslant K(\tau, L)$ следует $\pi^{\prime} \simeq_{\sigma} \pi^{\prime \prime}$. Для подтверждения равносильности $K(\tau(\sigma), L(\sigma))$ и $K(\tau, L)$ достаточно убедиться в том, что $\pi^{\prime}$ и $\pi^{\prime \prime}$ эквивалентны в модели $K(\tau(\sigma), L(\sigma))$. Выберем произвольное начальное состояние памяти $\xi$ из множества $\Sigma_{0}$. Если одна из рассматриваемых схем (например, $\pi^{\prime}$ ) имеет успешное вычисление на функции разметки $\mu_{\xi}$, то программа $\pi^{\prime}$ в семантике $\sigma$ также имеет успешное вычисление на $\xi$. Поскольку $\pi^{\prime} \simeq_{\sigma} \pi^{\prime \prime}$, вычисление $\pi^{\prime \prime}$ в семантике $\sigma$ на начальном состоянии $\xi$ имеет тот же результат, что и вычисление $\pi^{\prime}$. Это означает, что значение $F_{\pi^{\prime}}\left(\mu_{\xi}\right)$ определено.

Предположим, что $F_{\pi^{\prime}}\left(\mu_{\xi}\right)^{\prime}=h_{1}=s_{1} s_{2} \ldots s_{k}$ и $F_{\pi}\left(\mu_{\xi}\right)=h_{2}$. Ввиду того, что $\sigma-$ обратимая абстрактная семантика, для о. ц. $h_{1}=s_{1} s_{2} \ldots s_{k}$ существует о.ц. $h_{1}^{-1}=$ 
$s_{k}^{-1} \ldots s_{2}^{-1} s_{1}^{-1}$, задающая в семантике $\sigma$ преобразование, обратное $I h_{1}$, т. е. $I h_{1}^{-1}$ 。 $I h_{1}(\zeta)=I h_{1} h_{1}^{-1}(\zeta)=\zeta$ для любого состояния памяти $\zeta$ из $\Sigma$. Согласно определению стандартной аппроксимации это означает, что в модели $K(\tau(\sigma), L(\sigma))$ выполняются соотношения $h_{1} h_{1}^{-1} \tau(\sigma) e$ и $h_{1} h_{1}^{-1} h \tau(\sigma) h$, где в качестве $h$ может выступать любая о. ц. из $S^{*}$. Поэтому для каждого префикса о. ц. $h_{1}$ и $h_{2}$ в о. ц. $H=h_{1} h_{1}^{-1} h_{2}$ имеется $\tau(\sigma)$-эквивалентный префикс, и поведение всякой $\tau(\sigma)$-согласованной функции разметки на о. ц. $H$ однозначно определяет ее поведение на о. ц. $h_{1}$ и $h_{2}$, т. е. $\mu_{1} \stackrel{H}{=} \mu_{2}$ влечет $\mu_{1} \stackrel{h_{1}}{=} \mu_{2}$ и $\mu_{1} \stackrel{h_{2}}{=} \mu_{2}$. Поскольку $[L]=[L(\sigma)]$, множество $L$ содержит функцию разметки $\mu$ такую, что $\mu \stackrel{H}{=} \mu_{\xi}$. Отсюда, учитывая равенство $\tau=\tau(\sigma)$, эквивалентность схем $\pi^{\prime} \simeq_{\tau, L} \pi^{\prime \prime}$ и следствие 1 , получаем, что

$$
h_{1}=F \pi^{\prime}\left(\mu_{\xi}\right)=F_{\pi^{\prime}}(\mu) \tau(\sigma) F \pi^{\prime \prime}(\mu)=F_{\pi^{\prime \prime}}\left(\mu_{\xi}\right)=h_{2} .
$$

Значит схемы $\pi^{\prime}$ и $\pi^{\prime \prime}$ эквивалентны в модели $K(\tau(\sigma), L(\sigma))$, и $K(\tau, L)$ равносильна стандартной аппроксимации $K(\tau(\sigma), L(\sigma))$ обратимой семантики $\sigma$.

Следствие 5. Если $\sigma$ - обратимая абстрактная семантика, то стандартная аппроксимация $K(\tau(\sigma), L(\sigma))$ является наилучшим приближением $\sigma$ в классе согласованных формальных моделей.

\section{5. Абстрактные семантики, не имеющие наилучшей формальной аппроксимации}

Обратимся к исследованию абстрактных семантик, имеющих нестандартные наилучшие и минимальные приближения в классе всевозможных формальных моделей. Используемый здесь метод построения подобных семантик основан на следующей идее. Если параметры $\tau$ и $\tau^{\prime}$ правоконгруэнтных моделей $K(\tau, L)$ и $K\left(\tau^{\prime}, L^{\prime}\right)$ подчиняются условию $\tau \rightarrow \tau^{\prime}$, то стандартной аппроксимацией прямой суммы семантик $\sigma(\tau, L)$ и $\sigma\left(\tau^{\prime}, L^{\prime}\right)$ служит модель $K\left(\tau, L \cup L^{\prime}\right)$. Однако, в случае, когда для всякой пары схем $\pi_{1}$ и $\pi_{2}$ эквивалентность $\pi_{1} \simeq_{\tau, L} \pi_{2}$ влечет $\pi_{1} \simeq_{\tau^{\prime}, L^{\prime}} \pi_{2}$, формальная модель $K(\tau, L)$ будет более точным приближением семантики $\sigma(\tau, L) \oplus \sigma\left(\tau^{\prime}, L^{\prime}\right)$, нежели стандартная аппроксимация $K\left(\tau, L \cup L^{\prime}\right)$. Поэтому сначала мы обоснуем существование моделей $K(\tau, L)$ и $K\left(\tau^{\prime}, L^{\prime}\right)$, обладающих указанными свойствами.

В качестве символьного базиса возьмем множества $S=\left\{s_{1}, s_{2}\right\}$ и $P=\{p\}$. Обозначим через $h_{1, n}$ о. ц. $s_{1}\left(s_{1}\right)^{n !}\left(s_{2}\right)^{n !} s_{2}$, а через $h_{2, n}$ о. ц. $s_{2}\left(s_{2}\right)^{n !}\left(s_{1}\right)^{n !} s_{1}, n \geqslant 0$, полагая при этом $0 !=0$. Цепочку $H$ будем называть составной, если $H$ представима в виде $H=h_{i, n} h, i \in\{1,2\}, n \geqslant 0$, где $h$ - некоторая (возможно пустая) о. ц. из $S^{*}$. В противном случае $H$ будем называть протой о. ц. Отметим, что составная цепочка $H$ допускает единственное разбиение указанного вида.

Правая конгруэнция $\tau$ на $S^{*}$ задается системой определяющих соотношений

(1) $h_{1,2 m} \tau h_{1,2 n} \tau h_{2,0}, n \geqslant 1, m \geqslant 1$;

(2) $h_{2,2 m} \tau h_{2,2 n} \tau h_{1,0}, n \geqslant 1, m \geqslant 1$;

(3) $h_{1,2 n-1} \tau h_{2,2 n-1}, n \geqslant 1$.

Правая конгруэнция $\tau^{\prime}$ на $S^{*}$ задается определяющими соотношениями 
(1') $h_{i, 2 n} \tau^{\prime} h_{j, 2 m}, i, j \in\{0,1\}, n \geqslant 0, m \geqslant 0$;

$\left(2^{\prime}\right) h_{1,2 n-1} \tau^{\prime} h_{2,2 n-1}, n \geqslant 1$.

Исходя из определений $\tau$ и $\tau^{\prime}$, нетрудно видеть, что $\tau \rightarrow \tau^{\prime}$, причем простые о. ц. эквивалентны относительно $\tau$ и $\tau^{\prime}$ тогда и только тогда, когда они тождественно совпадают. Если цепочка $H^{\prime}$ эквивалентна составной о. ц. $H=h_{i, n} h$ относительно $\tau$ или $\tau^{\prime}$, то $H^{\prime}$ представима в виде $H^{\prime}=h_{j, m} h$ и при этом $h_{i, n}$ и $h_{j, m}$ подчиняются соотношениям 1-3 или $1^{\prime}, 2^{\prime}$ соответственно.

Мы будем рассматривать следующие семейства функций разметки :

$\mu_{1,2 n}, n \geqslant 1$, - функции разметки, принимающие значение 1 только на о.ц. $s_{1}\left(s_{1}\right)^{(2 n) !}, s_{1}\left(s_{1}\right)^{(2 n) !}\left(s_{2}\right)^{(2 n) !}$ и $s_{2}$;

$\mu_{2,2 n}, n \geqslant 1$, - функции разметки, принимающие значение 1 только на о.ц. $s_{2}\left(s_{2}\right)^{(2 n) !}, s_{2}\left(s_{2}\right)^{(2 n) !}\left(s_{1}\right)^{(2 n) !}$ и $s_{1}$;

$\mu_{2 n-1}, n \geqslant 1$, - функции разметки, принимающие значение 1 только на о.ц. $s_{1}\left(s_{1}\right)^{(2 n-1) !}, s_{1}\left(s_{1}\right)^{(2 n-1) !}\left(s_{2}\right)^{(2 n-1) !}, s_{2}\left(s_{2}\right)^{(2 n-1) !}$ и $s_{2}\left(s_{2}\right)^{(2 n-1) !}\left(s_{1}\right)^{(2 n-1) !}$;

$\lambda$ - функцию разметки, обращающуюся в 1 только на о.ц. $s_{1}$ и $s_{2}$.

Все вышеуказанные функции разметки согласованы с отношениями эквивалентности $\tau$ и $\tau^{\prime}$, так как эти функции придают л.п. $p$ значение 1 только на простых о.ц. Кроме того все описанные здесь функции разметки имеют одинаковое поведение на пустой о.ц., и поэтому для каждой схемы $\pi$ результаты всех вычислений, порождаемых этими функциями, начинаются одним и тем же о.с. $s_{1}$ или $s_{2}$ в зависимости от вида схемы. Обозначим через $L_{0}$ множество, образованное всеми функциями разметки, относящимися к первым трем семействам, т.е. $L_{0}=\left\{\mu_{i, 2 n}: i \in\{1,2\}, n \geqslant 1\right\} \cup\left\{\mu_{2 n-1}: n \geqslant 1\right\}$.

Лемма 1. Каковы бы ни были схемы $\pi_{1}$ и $\pi_{2}$ над символьным базисом $(S, P)$, если $\pi_{1} \simeq_{\tau, L_{0}} \pi_{2}$, mo $\pi_{1} \simeq_{\tau^{\prime},\{\lambda\}} \pi_{2}$.

Доказателъство. Пусть $\pi_{1} \simeq_{\tau, L_{0}} \pi_{2}$ и $F_{\pi_{1}}(\lambda)=H_{1}$. Покажем, что вычисление схемы $\pi_{2}$ на функции разметки $\lambda$ завершается с результатом $H_{2}, H_{2} \tau^{\prime} H_{1}$.

Если $H_{1}$ - пустая о. ц. $e$, то из $\lambda \stackrel{e}{=} \mu_{1}, \pi_{1} \simeq_{\tau, L_{0}} \pi_{2}$ и $\tau \rightarrow \tau^{\prime}$ следует, что $H_{1}=F_{\pi_{1}}(\lambda)=F_{\pi_{1}}\left(\mu_{1}\right) \tau F_{\pi_{2}}\left(\mu_{1}\right)=F_{\pi_{2}}(\lambda)$ и $H_{1} \tau^{\prime} F_{\pi_{2}}(\lambda)=H_{2}$.

Предположим, что цепочка $H_{1}$ начинается о. с. $s_{1}$. Поскольку определения эквивалентностей $\tau$ и $\tau^{\prime}$, а также рассматриваемых функций разметки симметричны относительно о.с. $s_{1}$ и $s_{2}$, все последующие рассуждения применимы и в случае, когда $H_{1}$ начинается о. с. $s_{2}$.

Если $H_{1}$ - простая о. ц., то, как и в случае $H_{1}=e$, из условия эквивалентности простых цепочек, $\lambda \stackrel{H_{1}}{=} \mu_{2,2}, \pi_{1} \simeq_{\tau, L_{0}} \pi_{2}$ и $\tau \rightarrow \tau^{\prime}$ следует $H_{1} \tau F_{\pi_{2}}(\lambda)=H_{2}$.

Если $H_{1}$ - составная о. ., то $H_{1}$ представима в одном из следующих видов: $H_{1}=h_{1,2 k-1} h, k \geqslant 1, H_{1}=h_{1,2 k} h, k \geqslant 1$, или $H_{1}=h_{1,0} h$, где $h-$ некоторая цепочка из $S^{*}$. Исследуем каждый из возможных вариантов $H_{1}$.

1. $H_{1}=h_{1,2 k-1} h, k \geqslant 1$, Тогда $\lambda \stackrel{H_{1}}{=} \mu_{2,2 n}$ и $H_{1}=F_{\pi_{1}}(\lambda)=F_{\pi_{1}}\left(\mu_{2,2 n}\right)$ при любом $n \geqslant 1$. Учитывая $\pi_{1} \simeq_{\tau, L_{0}} \pi_{2}$, можно заключить, что $H_{1} \tau F_{\pi_{2}}\left(\mu_{2,2 n}\right)=H^{\prime}$. Как следует из определения правой конгруэнции $\tau$, цепочка $H^{\prime}$ представима либо в виде $H^{\prime}=H_{1}$, либо в виде $H^{\prime}=h_{2,2 k-1} h$. 
Если $H^{\prime}=H_{1}$, то $\lambda \stackrel{H_{1}}{=} \mu_{2,2 n}$ приводит к $F_{\pi_{2}}(\lambda)=F_{\pi_{2}}\left(\mu_{2,2 n}\right)=H_{1}$.

Рассмотрим случай $H^{\prime}=h_{2,2 k-1} h$. Учитывая $\left(\tau, L_{0}\right)$-эквивалентность схем $\pi_{1}$ и $\pi_{2}$ и то обстоятельство, что $H_{1}=F_{\pi_{1}}\left(\mu_{2,2 n}\right) \tau F_{\pi_{2}}\left(\mu_{2,2 n}\right)=H^{\prime}$ при любых $n \geqslant 1$, а $\mu_{2,2 m} \stackrel{H^{\prime}}{=} \mu_{2 m-1}$ при любых $m^{\prime} \geqslant\left|H^{\prime}\right|$, можно заключить, что при достаточно больших $m$ выполняется $H^{\prime}=F_{\pi_{2}}\left(\mu_{2,2 m}\right)=F_{\pi_{2}}\left(\mu_{2 m-1}\right) \tau F_{\pi_{1}}\left(\mu_{2 m-1}\right)$. Коль скоро все о. ц., являющиеся значениями функционала $F_{\pi_{1}}$ на функциях разметки $\mu \in L_{0}$, начинаются $s_{1}$, то $F_{\pi_{1}}\left(\mu_{2 m-1}\right)=H_{1}$ при любом $m \geqslant\left|H^{\prime}\right|=\left|H_{1}\right|$. При $m \geqslant\left|H_{1}\right|$, функции разметки $\mu_{1,2 m}$ и $\mu_{2 m-1}$ имеют одинаковое поведение на $H_{1} ;$ поэтому из $\pi_{1} \simeq_{\tau, L_{0}} \pi_{2}$ следует $H_{1}=F_{\pi_{1}}\left(\mu_{2 m-1}\right)=F_{\pi_{1}}\left(\mu_{1,2 m}\right) \tau F_{\pi_{2}}\left(\mu_{1,2 m}\right)$. Так как $H^{\prime}$ начинается о. с. $s_{2}$, то $F_{\pi_{2}}\left(\mu_{1,2 m}\right)=H^{\prime}$. На цепочках, начинающихся о.с. $s_{2}$, функции разметки $\mu_{1,2 m}$ и $\lambda$ имеют одинаковое поведение; отсюда следует $F_{\pi_{2}}(\lambda)=F_{\pi_{2}}\left(\mu_{1,2 m}\right)=H^{\prime} \tau H_{1}$ и $F_{\pi_{2}}(\lambda) \tau^{\prime} F_{\pi_{1}}(\lambda)=H_{1}$

2. $H_{1}=h_{1,2 k} h, k \geqslant 1$. Тогда $\lambda \stackrel{H_{1}}{=} \mu_{2,2 n}$ и $\pi_{1} \simeq_{\tau, L_{0}} \pi_{2}$ влечет $F_{\pi_{2}}\left(\mu_{2,2 n}\right)=H_{n}^{\prime} \tau H_{1}$ при любом $n \geqslant 1$. Из определения конгруэнции $\tau$ следует, что $H_{n}^{\prime}$ представима лиибо в виде $H_{n}^{\prime}=h_{1,2 n} h$, либо в виде $H_{n}^{\prime}=h_{2,0} h$.

В первом случае совпадение поведения функций разметки $\lambda$ и $\mu_{2,2 n}$ на цепочках, начинающихся о. с. $s_{1}$, обеспечивает $F_{\pi_{2}}(\lambda)=F_{\pi_{2}}\left(\mu_{2,2 n}\right)=H_{n}^{\prime} \tau H_{1}$ и $F_{\pi_{1}}(\lambda) \tau^{\prime} F_{\pi_{2}}(\lambda)$.

Во втором случае равенство $F_{\pi_{2}}\left(\mu_{2,2 n}\right)=h_{2,0} h=H_{n}^{\prime}$ будет выполняться при любом значении $n \geqslant 1$, ибо на о. ц. $H_{n}^{\prime}$ все функции разметки $\mu_{2,2 n}, n \geqslant 1$, имеют одинаковое поведение, совпадающее с поведением функций $\mu_{2 n-1}$. Учитывая эквивалентность схем $\pi_{1}$ и $\pi_{2}$, получаем $H_{n}^{\prime}=F_{\pi_{2}}\left(\mu_{2,2 n}\right)=F_{\pi_{2}}\left(\mu_{2 n-1}\right) \tau F_{\pi_{1}}\left(\mu_{2 n-1}\right)=H_{n}^{\prime \prime}$ для каждого $n \geqslant 1$. Так как все о. ц. $H_{n}^{\prime \prime}$ начинаются о.с. $s_{1}$ и $\tau$-эквивалентны $h_{2,0} h$, они неизбежно имеют вид $H_{n}^{\prime \prime}=h_{1,2 i_{n}} h$. Далее надлежит обратить внимание на то, что при порождении о. ц. $h_{1,2 i_{n}} h$ в процессе вычисления схемы $\pi_{1}$ на функциях разметки $\mu_{2 n-1}$ л.п. $p$ лишь однажды на $(1+(2 n-1)$ !)-м этапе принимает значение 1 , и поэтому $\pi_{1}$ на функциях разметки $\mu_{2 n-1}$ не может завершать вычисления, строя при этом сколь угодно длинные цепочки вида $s_{1}\left(s_{1}\right)^{(2 n) !}\left(s_{2}\right)^{(2 n) !} s_{2}$. Таким образом, функционал $F_{\pi_{1}}$ на множестве функций разметки $\left\{\mu_{2 n-1}: n \geqslant 1\right\}$ имеет лишь конечное число различных значений. Обозначим через $N$ длину самой протяженной цепочки $H_{n}^{\prime \prime}$. Тогда на всех о. ц. $H_{n}^{\prime \prime}$ функции разметки $\mu_{2 m-1}$ и $\mu_{1,2 m}$ при $m>N$ имеют одинаковое поведение (всегда придают $p$ значение 0 ), что влечет $H_{m}^{\prime \prime}=F_{\pi_{1}}\left(\mu_{2 m-1}\right)=F_{\pi_{1}}\left(\mu_{1,2 m}\right) \tau F_{\pi_{2}}\left(\mu_{1,2 m}\right)$. Ввиду того, что все о. ц., порождаемые схемой $\pi_{2}$ на функциях разметки из $L_{0}$, начинаются символом $s_{2}$, поведение функций $\mu_{1,2 m}$ и $\lambda$ на этих цепочках будет одинаковым, и $F_{\pi_{2}}\left(\mu_{1,2 m}\right)=F_{\pi_{2}}(\lambda)$. В итоге получаем $H_{1} \tau H_{n}^{\prime} \tau H_{m}^{\prime \prime} \tau F_{\pi_{2}}(\lambda)=H_{2}$ и $F_{\pi_{1}}(\lambda) \tau^{\prime} F_{\pi_{2}}(\lambda)$.

3. $H_{1}=h_{1,0} h$. Тогда из $\lambda \stackrel{H_{1}}{=} \mu_{2,2 n}$ и $\pi_{1} \simeq_{\tau, L_{0}} \pi_{2}$ следует, ч'го $H_{1}=F_{\pi_{1}}(\lambda)=$ $F_{\pi_{1}}\left(\mu_{2,2 n}\right) \tau F_{\pi_{2}}\left(\mu_{2,2 n}\right)=H_{n}^{\prime}$ для любого $n \geqslant 1$. Если $H_{n}^{\prime}=H_{1}$, то $F_{\pi_{2}}(\lambda)=H_{1}$. Предположим, что все о. ц. $H_{n}^{\prime}$ имеют вид $h_{2,2 i_{n}} h$, где $n \geqslant 1, i_{n} \geqslant 1$. Рассмотрим множество цепочек $G=\left\{H_{n}^{\prime}: n \geqslant 1\right\}$ и в зависимости от его мощности исследуем отдельно два различных варианта функционирования схем $\pi_{1}$ и $\pi_{2}$.

3.1. $G$ - конечное множество. Обозначим через $H_{m}^{\prime}$ самую протяженную о. ц. множества $G$. Тогда при любом $n>\left|H_{m}^{\prime}\right|$ функции разметки $\mu_{2,2 n}$ и $\mu_{2 n-1}$ имеют одинаковое поведение на о. ц. $H_{m}^{\prime}$, что влечет соотношения $H_{m}^{\prime}=F_{\pi_{2}}\left(\mu_{2,2 n}\right)=$ $F_{\pi_{2}}\left(\mu_{2 n-1}\right) \tau F_{\pi_{1}}\left(\mu_{2 n-1}\right)$. Цепочки, порождаемые $\pi_{1}$ на функциях разметки из $L_{0}$, начинаются о.с. $s_{1}$, и поэтому $F_{\pi_{1}}\left(\mu_{2 n-1}\right)=H_{1}$ при любом $n>\left|H_{m}^{\prime}\right|$. Так как $\mu_{2 n-1} \stackrel{H_{1}}{=} \mu_{1,2 n}, H_{1}=F_{\pi_{1}}\left(\mu_{2 n-1}\right)=F_{\pi_{1}}\left(\mu_{1,2 n}\right) \tau F_{\pi_{2}}\left(\mu_{1,2 n}\right)$. Коль скоро о. ц, порождаемые схемой $\pi_{2}$ на функциях разметки из $L_{0}$ начинаются о. с. $s_{2}$, функции $\mu_{1,2 n}$ 
и $\lambda$ имеют на них одинаковое поведение, и поэтому $F_{\pi_{2}}\left(\mu_{1,2 n}\right)=F_{\pi_{2}}(\lambda)$. В итоге получаем, что $H_{1} \tau^{\prime} F_{\pi_{2}}(\lambda)$.

3.2. Множество $G$ содержит бесконечно много различных цепочек. Тогда для бесконечно большого числа функций разметки $\mu_{2,2 n}$ л. п. $p$ дважды принимает значение 1 в процессе вычисления схемы $\pi_{2}$ на $\mu_{2,2 n}$. Рассмотрим вычислительную трассу схемы $\pi_{2}$ на одной из таких функций разметки $\mu_{2,2 n}$, где $n$ превосходит общее число преобразователей схемы $\pi_{2}$. На протяжении первых $1+(2 n)$ ! этапов вычисления значение л. п. $p$ равно 0 , и трасса проходит через преобразователи, помеченные о. с. $S_{2}$. Это приводит к тому, что спустя некоторое количество $r_{1}, r_{1}<n$, начальных этапов будет достигнут преобразователь $\nu_{1}$, который далее посещается периодически по циклу $C_{1}$ через каждые $t_{1}, t_{1}<n$, тактов вычисления до тех пор, пока $p$ не примет значение 1 после прохождения вершины $\nu_{1}$ на $(2+(2 n)$ !)-м этапе вычисления. Таким образом, для некоторого натурального $i_{1}$ имеет место равенство $r_{1}+i_{1} t_{1}=(2 n) !+1$. Последующие $1+(2 n)$ ! шагов вычисления протекают по аналогичному сценарию: спустя $r_{2}, r_{2}<n$, этапов вычисление, выйдя из преобразователя $\nu_{1}$, попадает в цикл $C_{2}$, содержащий $t_{2}, t_{2}<n$, преобразователей с о.с. $s_{1}$, который будет покинут после того, как на $2(2+(2 n)$ !)-м шаге функция разметки $\mu_{2,2 n}$ придаст л. п. $p$ значение 1 . Далее значение $p$ вновь становится равным 0 , и вычисление за ограниченное число шагов порождает о. ц. $h$, достигая при этом выхода схемы $\pi_{2}$.

Сопоставим теперь рассмотренное вычисление с функционированием схемы $\pi_{2}$ на функции разметки $\mu_{2 n-1}$. На цепочках длины $(2 n-1)$ !, начинающихся о. с. $s_{2}$, функции $\mu_{2 n-1}$ и $\mu_{2,2 n}$ имеют одинаковое поведение. Поэтому вычисление $\pi_{2}$ на $\mu_{2 n-1}$ на $r_{1}$-м шаге также достигает преобразователя $\nu_{1}$ и попадает в цикл $C_{1}$, обход которого совершается до тех пор, пока $p$ не примет значение 1 . Так как длина $t_{1}$ цикла $C_{1}$ является делителем числа $n$ !, то для некоторого натурального $j_{1}$ справедливо равенство $\left(i_{1}-j_{1}\right) t_{1}=(2 n) !-(2 n-1)$ !. Отсюда следует $r_{1}+j_{1} t_{1}=(2 n-1) !+1$. Это означает, что в процессе данного вычисления совершается $j_{1}$ полных обходов цикла $C_{1}$, и л. п. $p$ изменяет значение с 0 на 1 на $(2+(2 n-1)$ !)-м этапе после прохождения через преобразователь $\nu_{1}$. Прохождение цикла $C_{2}$ происходит совершенно аналогично. Таким образом, вычисление схемы $\pi_{2}$ на функции разметки $\mu_{2 n-1}$ повторяет вычисление $\pi_{2}$ на функции $\mu_{2,2 n}$ с той лишь разницей, что циклы $C_{1}$ и $C_{2}$ обходятся меньшее число раз. Нетрудно видеть, что о.ц., порожденная таким вычислением, будет иметь вид $h_{2,2 n-1} h$. Это означает, что схема $\pi_{2}$ на множестве функций разметки $\left\{\mu_{2 n-1}: n \geqslant 1\right\}$ порождает бесконечно много различных о. . вида $h_{2,2 n-1} h$. Тогда схема $\pi_{1}$ в силу эквивалентности $\pi_{1} \simeq_{\tau, L_{0}} \pi_{2}$ порождает на том же множестве функций разметки бесконечно большое число различных о.ц. вида $h_{1,2 n-1} h$. Проанализировав и сопоставив вычисления $\pi_{1}$ на функциях разметки $\mu_{2 n-1}$ и $\mu_{1,2 n}$, подобно тому, как это было сделано выше для схемы $\pi_{2}$, можно заключить, что на функциях разметқи $\mu_{1,2 n}, n \geqslant 1$, функционал $F_{\pi_{1}}$ принимает бесконечно много различных значений вида $h_{1,2 n} h$. Учитывая $\tau$-эквивалентность схем $\pi_{1}$ и $\pi_{2}$, а также то обстоятельство, что все цепочки, порождаемые схемой $\pi_{2}$ на функциях разметки из $L_{0}$, начинаются о.c. $s_{2}$, заключаем, что все вычисления схемы $\pi_{2}$ на функциях разметки $\mu_{1,2 n}, n \geqslant 1$, порождают одну и ту же о.ц. $h_{2,0} h$. Поскольку на этой цепочке поведение функций разметки $\mu_{1,2 n}$ и $\lambda$ совпадают, $H_{2}=F_{\pi_{2}}(\lambda)=F_{\pi_{2}}\left(\mu_{1,2 n}\right)=h_{2,0} h$. Таким образом, в этом случае справедливы соотношения $F_{\pi_{1}}(\lambda)=H_{1}=h_{1,0} h \tau^{\prime} h_{2,0}=H_{2}=F_{\pi_{2}}(\lambda)$.

Итак, если схемы $\pi_{1}$ и $\pi_{2}$ эквивалентны в модели $K\left(\tau, L_{0}\right)$ и $F_{\pi_{2}}(\lambda)=H_{1}$, вы- 
числение схемы $\pi_{2}$ на функции разметки $\lambda$ завершается построением о.ц. $H_{2}, \tau^{\prime}$ эквивалентной цепочке $H_{1}$. Приведенные выше рассуждения инвариантны относительно выбора схемы $\pi_{1}$ или $\pi_{2}$, и поэтому эквивалентность $\pi_{1} \simeq_{\tau, L_{0}} \pi_{2}$ влечет эквивалентность $\pi_{1} \simeq_{\tau^{\prime},\{\lambda\}} \pi_{2}$.

Утверждение леммы используется при доказательстве следующих теорем.

Теорема 6. Существует абстрактная семантика $\sigma$, стандартная аппроксимация $K(\tau(\sigma), L(\sigma))$ которой не является минимальным приближением $\sigma$ в классе правоконгруэнтных моделей.

Доказательство. Рассмотрим отношения эквивалентности $\tau$ и $\tau^{\prime}$, а также множества функций разметки $L_{0}$ и $\{\lambda\}$, фигурирующие в лемме 1 . Положим $L=L_{0} \cup\{\lambda\}$. Покажем, что $\sigma\left(\tau, L_{0}\right) \oplus \sigma\left(\tau^{\prime},\{\lambda\}\right)$ - искомая абстрактная семантика $\sigma$ над символьным базисом $S=\left\{s_{1}, s_{2}\right\}$ и $P=\{p\}$.

Поскольку $\tau \rightarrow \tau^{\prime}$, стандартная аппроксимация указанной семантики $\sigma$ имеет вид $K(\tau(\sigma), L(\sigma))=K(\tau, L)$. Проведем сравнительный анализ выразительных возможностей формальных моделей $K(\tau, L)$ и $K\left(\tau, L_{0}\right)$.

Из $L_{0} \subset L$ и достаточного условия сравнимости формальных моделей (2) следует $K\left(\tau, L_{0}\right) \leqslant K(\tau, L)$. Рассмотрим пару схем $\Pi_{5}$ и $\Pi_{6}$, изображенных на рис. 3 . Нетрудно видеть, что на множестве $L$ функционалы $F_{\Pi_{5}}$ и $F_{\Pi_{6}}$ принимают следующие значения: $F_{\Pi_{5}}\left(\mu_{1,2 m}\right)=h_{1,2 m}, F_{\Pi_{5}}\left(\mu_{2 m-1}\right)=h_{1,2 m-1}, F_{\Pi_{5}}\left(\mu_{2,2 m}\right)=F_{\Pi_{5}}(\lambda)=h_{1,0}$, $F_{\Pi_{6}}\left(\mu_{2,2 m}\right)=h_{2,2 m}, F_{\Pi_{6}}\left(\mu_{2 m-1}\right)=h_{2,2 m-1}, F_{\Pi_{6}}\left(\mu_{1,2 m}\right)=F_{\Pi_{6}}(\lambda)=h_{2,0}, m \geqslant 1$. Для любой функции разметки $\mu \in L_{0}$ выполняется соотношение $F_{\Pi_{5}}(\mu) \tau F_{\Pi_{6}}(\mu)$, и поэтому $\Pi_{5} \simeq_{\tau, L_{0}} \Pi_{6}$. В то же время на функции разметки $\lambda$ схемы $\Pi_{5}$ и $\Pi_{6}$ порождают о. ц. $h_{1,0}$ и $h_{2,0}$ соответственно, принадлежащие разным классам $\tau$-эквивалентности на $S^{*}$. Таким образом, модели $K\left(\tau, L_{0}\right)$ и $K(\tau, L)$ неравносильны.

Теперь убедимся в том, что формальная модель $K\left(\tau, L_{0}\right)$ является наилучшей аппроксимацией абстрактной семантики $\sigma=\sigma\left(\tau, L_{0}\right) \oplus \sigma\left(\tau^{\prime},\{\lambda\}\right)$. Возьмем произвольную пару схем $\pi_{1}$ и $\pi_{2}$. Если $\pi_{1} \simeq_{\sigma} \pi_{2}$, то по определению прямой суммы абстрактных семантик выполняется соотношение $\pi_{1} \simeq_{\sigma\left(\tau, L_{0}\right)} \pi_{2}$, откуда следует $\pi_{1} \simeq_{\tau, L_{0}} \pi_{2}$. Если $\pi_{1} \simeq_{\tau, L_{0}} \pi_{2}$, то согласно лемме 1 выполняется соотношение $\pi_{1} \simeq_{\tau^{\prime},\{\lambda\}} \pi_{2}$. По определению абстрактных семантик, соответствующих формальным моделям $K\left(\tau, L_{0}\right)$ и $K\left(\tau^{\prime},\{\lambda\}\right)$, отсюда следует $\pi_{1} \simeq_{\sigma\left(\tau, L_{0}\right)} \pi_{2}$ и $\pi_{1} \simeq_{\sigma\left(\tau^{\prime},\{\lambda\}\right)} \pi_{2}$, что приводит в итоге к равносильности $\pi_{1} \simeq_{\sigma} \pi_{2} \Longleftrightarrow \pi_{1} \simeq_{\tau, L_{0}} \pi_{2}$.

В результате получаем соотношение

$$
\sigma \approx K\left(\tau, L_{0}\right)<K(\tau, L)=K(\tau(\sigma), L(\sigma))
$$

связывающее абстрактную семантику $\sigma=\sigma\left(\tau, L_{0}\right) \oplus \sigma\left(\tau^{\prime},\{\lambda\}\right)$ и формальные модели $K(\tau, L)$ и $K\left(\tau, L_{0}\right)$. Данное соотношение свидетельствует о том, что стандартная аппроксимация семантики $\sigma$ не является минимальным приближением $\sigma$ в классе правоконгруэнтных моделей.

Теорема 7. Существуют абстрактные семантики, не имеющие наилучшего приближения в классе всевозможных формалъных моделей.

Доказателъство. Рассмотрим символьный базис $S=\left\{s_{1}, s_{2}\right\}, P=\{p\}$ и построенные ранее отношения эквивалентности $\tau$ и $\tau^{\prime}$ на $S^{*}$, а также множества функций разметки $L_{0}$ и $\{\lambda\}$. Обозначим через $\nu_{0}$ функцию разметки, придающую л. п. 

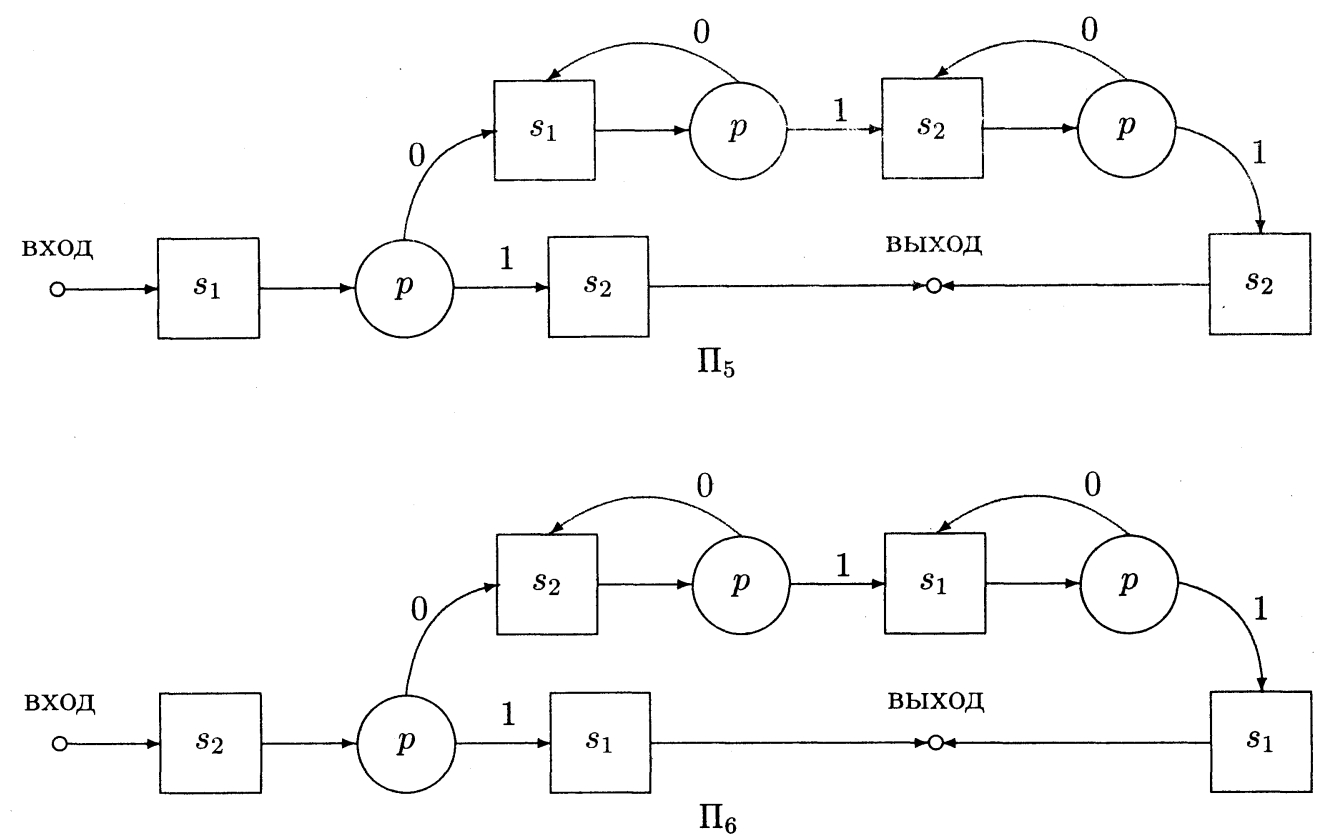

Рис. 3.

$p$ значение 0 на каждой о. ц., и покажем, что $\sigma=\sigma\left(\tau, \nu_{0}\right) \oplus \sigma\left(\tau^{\prime},\{\lambda\}\right)$ - искомая абстрактная семантика, не имеющая наилучшей формальной аппроксимации.

Вначале убедимся, что стандартная аппроксимация $K\left(\tau,\left\{\lambda, \nu_{0}\right\}\right)$ является минимальным приближением $\sigma$. Действительно, для произвольной формальной модели $K\left(\tau^{\prime \prime}, L^{\prime \prime}\right)$ из $\sigma \leqslant K\left(\tau^{\prime \prime}, L^{\prime \prime}\right) \leqslant K\left(\tau,\left\{\lambda, \nu_{0}\right\}\right)$ согласно предложениям 2 и 4 следует $\tau^{\prime \prime} \equiv \tau$ и $L^{\prime \prime} \subseteq\left[L^{\prime \prime}\right]=\left[\left\{\lambda, \nu_{0}\right\}\right]=\left\{\lambda, \nu_{0}, \nu_{1}, \nu_{2}\right\}$, где $\nu_{1}, i \in\{1,2\},-$ функции разметки, придающие л. п. $p$ значение 1 на одной единственной о. ц. $s_{1}$. Схемы $\Pi_{7}$ и $\Pi_{8}$, изображенные на рис. 4 , эквивалентны в формальной модели $K\left(\tau,\left\{\lambda, \nu_{0}\right\}\right)$ и абстрактной семантике $\sigma$, но их вычисления на функциях разметки $\nu_{i}, i \in\{1,2\}$, существенно отличаются друг от друга; $F_{\Pi_{7}}\left(\nu_{1}\right)=h_{1,1}, F_{\Pi_{8}}\left(\nu_{2}\right)=h_{2,1}$, а значения $F_{\Pi_{7}}\left(\nu_{2}\right)$ и $F_{\Pi_{8}}\left(\nu_{1}\right)$ неопределены. Значит $\nu_{1} \notin L^{\prime \prime}$ и $\nu_{2} \notin L^{\prime \prime}$. Поскольку $[\lambda]=\{\lambda\}$ и $\left[\nu_{0}\right]=\left\{\nu_{0}\right\}$, приходим к выводу, что $L^{\prime \prime}=\left\{\lambda, \nu_{0}\right\}$.

Рассмотрим далее множество функций разметки $L^{\prime}=L_{0} \cup\left\{\nu_{0}\right\}$ и правоконгруэнтную модель $K\left(\tau, L^{\prime}\right)$. Согласно лемме 1 для любых схем $\pi_{1}$ и $\pi_{2}$ эквивалентность $\pi_{1} \simeq_{\tau, L^{\prime}} \pi_{2}$ влечет $\pi_{1} \simeq_{\tau,\left\{\nu_{0}\right\}} \pi_{2}$ и $\pi_{1} \simeq_{\tau^{\prime},\{\lambda\}} \pi_{2}$. Следовательно, формальная модель $K\left(\tau, L^{\prime}\right)$ аппроксимирует семантику $\sigma$. Обе рассмотренные аппроксимации $K\left(\tau,\left\{\lambda, \nu_{0}\right\}\right)$ и $K\left(\tau, L^{\prime}\right)$ семантики $\sigma=\sigma\left(\tau,\left\{\nu_{0}\right\}\right) \oplus \sigma\left(\tau^{\prime},\{\lambda\}\right)$ несравнимы друг с другом по отношению $\leqslant$; схемы $\Pi_{5}$ и $\Pi_{6}$, изображенные на рис. 3 , эквивалентны в $K\left(\tau, L^{\prime}\right)$, но неэквивалентны в модели $K\left(\tau,\left\{\lambda, \nu_{0}\right\}\right)$, а схемы $\Pi_{7}$ и $\Pi_{8}$, представленные на рис. 4 , напротив, эквивалентны в $K\left(\tau,\left\{\lambda, \nu_{0}\right\}\right)$, но не являются таковыми в модели $K\left(\tau, L^{\prime}\right)$. Это означает, что построенная абстрактная семантика $\sigma$ не имеет наилучшего приближения среди всевозможных формальных моделей. 

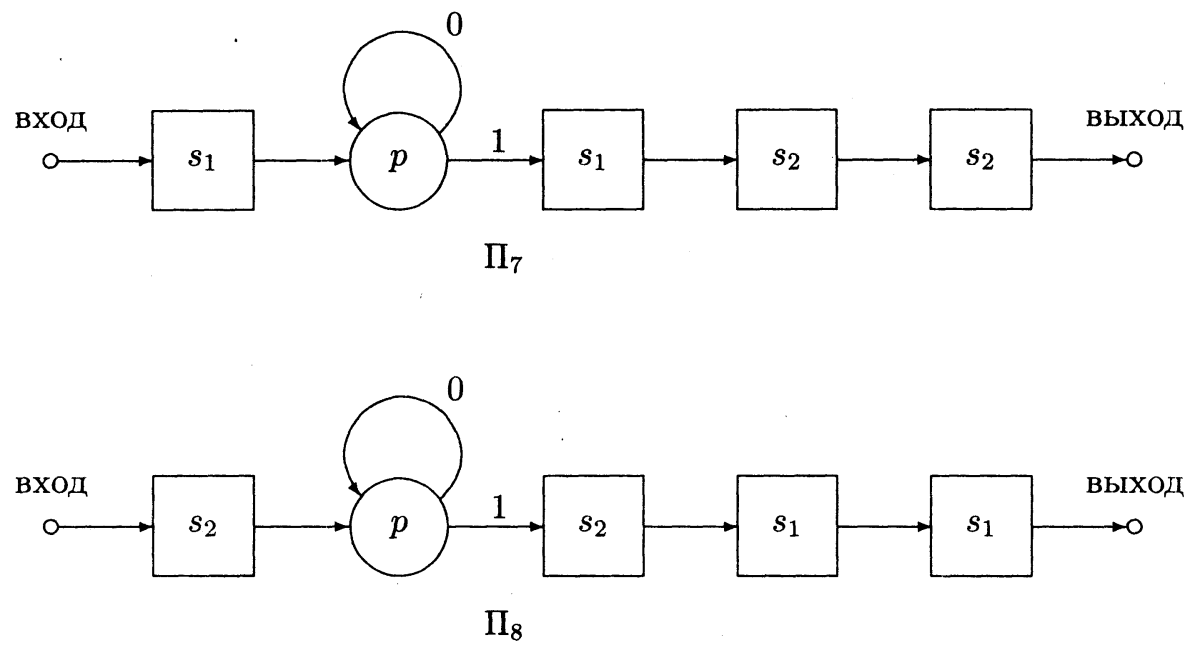

Puc. 4.

Следствие 6. Существуют абстрактные семантики, не имеющие равносилъных формальных моделей программ.

Теорема 8. Существуют согласованные формальные модели программ $K\left(\tau_{1}, L_{1}\right)$ и $K\left(\tau_{2}, L_{2}\right)$, классы равносилъности которых не имеют наименъшей верхней грани по отношению частичного порядка $\leqslant$.

Доказательство. Рассмотрим символьный базис $S=\left\{s_{1}, s_{2}\right\}, P=\{p\}$, отношения эквивалентности $\tau$ и $\tau^{\prime}$ на $S^{*}$, а также функции разметки $\lambda, \nu_{0}$, используемые при формулировке и доказательстве леммы 1 и теоремы 7. Согласно предложению 8 формальные модели $K\left(\tau,\left\{\nu_{0}\right\}\right)$ и $K\left(\tau^{\prime},\{\lambda\}\right)$ являются наилучшими аппроксимациями абстрактных семантик $\sigma\left(\tau,\left\{\nu_{0}\right\}\right)$ и $\sigma\left(\tau^{\prime},\{\lambda\}\right)$ соответственно. Так как при доказательстве теоремы 7 было установлено, что абстрактная семантика $\sigma\left(\tau,\left\{\nu_{0}\right\}\right) \oplus \sigma\left(\tau^{\prime},\{\lambda\}\right)$ не имеет наилучшей формальной аппроксимации в классе всевозможных моделей, то, согласно следствию из предложения 4, классы равносијьности $\sigma\left(\tau,\left\{\nu_{0}\right\}\right) / \approx$ и $\sigma\left(\tau^{\prime},\{\lambda\}\right) / \approx$ не имеют точной верхней грани по отношению частичного порядка $\leqslant$.

\section{6. Полугрупповые формальные модели и тотальные абстрактные семантики}

Предложение 9. Стандартная аппроксимация $K(\tau(\sigma), L(\sigma))$ тоталъной абстрактной семантики $\sigma$ является полугрупповой моделью.

Доказательство. Согласно предложению 5 стандартная аппроксимация семантики $\sigma=\langle\Sigma, \Sigma, I\rangle$ - правоконгруэнтная модель. По определению $\tau(\sigma)$ выполнимость $h_{1} \tau(\sigma) h_{2}$ и $h_{3} \tau(\sigma) h_{4}$ означает, что для любых $\xi^{\prime}$ и $\xi^{\prime \prime}$ из $\Sigma$ справедливы равенства 
$I h_{1}\left(\xi^{\prime}\right)=I h_{2}\left(\xi^{\prime}\right)$ и $I h_{3}\left(\xi^{\prime \prime}\right)=I h_{4}\left(\xi^{\prime \prime}\right)$. Отсюда следует, что равенство

$$
I h_{1} h_{3}(\xi)=I h_{3} \circ I h_{1}(\xi) I h_{4} \circ I h_{2}(\xi)=I h_{2} h_{4}(\xi)
$$

имеет место для любого состояния памяти $\xi \in \Sigma$, и поэтому $h_{1} h_{3} \tau(\sigma) h_{2} h_{4}$. Так как $L(\sigma)$ состоит из всевозможных функций разметки $\mu_{\xi}, \xi \in \Sigma$, значения которых определяются соотношением $\mu_{\xi} h(p)=I p(I h(\xi))$, для каждой л. п. $p \in P$ и цепочки $h \in S^{*}$, для любого о. с. $s \in S$ справедливо соотношение $\mu_{s}=\mu_{I s(\xi)} \in L(\sigma)$.

Теорема 9. Каждая полугрупповая модель $K(\tau, L)$ имеет равносильную тотальную абстрактную семантику $\sigma$.

Доказательство. Рассмотрим семантику $\sigma=\langle\Sigma, \Sigma, I\rangle$, область памяти $\Sigma$ и интерпретация символьного базиса $I$ которой определены так же, как для частичной семантики $\sigma(\tau, L)$ при доказательстве теоремы 2 , т. е.

$$
\begin{aligned}
\Sigma & =\left\{\xi_{\mu[h]}: \mu \in L,[h] \in S^{*} / \tau\right\}, \\
I s\left(\xi_{\mu[h]}\right) & =\xi_{\mu[h s]} \text { для каждого } s \in S \text { и } \xi_{\mu[h]} \in \Sigma, \\
I p\left(\xi_{\mu[h]}\right) & =\mu h(p) \text { для каждого } p \in P \text { и } \xi_{\mu[h]} \in \Sigma,
\end{aligned}
$$

где $[h]$ - класс $\tau$-эквивалентности, содержащий о. ц. $h$. Поскольку $K(\tau, L)$ обладает свойствами правоконгруэнтной модели, приведенное определение $\sigma$ корректно. Покажем, что для любых схем $\pi^{\prime}$ и $\pi^{\prime \prime}$ справедливо $\pi^{\prime} \simeq_{\tau, L} \pi^{\prime \prime} \Longleftrightarrow \pi^{\prime} \simeq_{\sigma} \pi^{\prime \prime}$.

Для каждой о.ц. $h$ и функции разметки $\mu$ обозначим через $\mu_{h}$ функцию разметки, значения которой на всякой о.ц. $H$ определяются соотношением $\mu_{h} H=\mu h H$. Множество $L$ замкнуто относительно операторного сдвига, поэтому $\left\{\mu_{h}: \mu \in L, h \in S^{*}\right\}=L$. Кроме того, для произвольной о. ц. $h$, функции разметки $\mu \in L$ и схемы $\pi$ индукцией по длине вычисления можно показать, что трасса вычисления программы $\pi$ в семантике $\sigma$ на начальном состоянии памяти $\xi_{\mu[h]}$ совпадает с трассой вычисления $\pi$ в модели $K(\tau, L)$ на функции разметки $\mu_{h}$.

Предположим, что $\pi^{\prime} \simeq_{\tau, L} \pi^{\prime \prime}$ и $\Phi_{\pi^{\prime}, \sigma}\left(\xi_{\mu[h]}\right)=\xi_{\mu[H]}$. Тогда на функции разметки $\mu_{h} \in L$ схемы $\pi^{\prime}$ и $\pi^{\prime \prime}$ строят о. ц. $h^{\prime}$ и $h^{\prime \prime}$ соответственно такие, что $h h^{\prime} \tau H$ и $h^{\prime} \tau h^{\prime \prime}$. Отношение $\tau$ является конгруэнцией, и поэтому справедливы соотношения $h h^{\prime} \tau h h^{\prime \prime}$ и $\Phi_{\pi, \sigma}\left(\xi_{\mu[h]}\right)=\xi_{\mu\left[h h^{\prime \prime}\right]}=\xi_{\mu[H]}$.

Если $\pi^{\prime} \simeq_{\sigma} \pi^{\prime \prime}$ и $F_{\pi^{\prime}}(\mu)=H$, то $\Phi_{\pi^{\prime}, \sigma}\left(\xi_{\mu[e]}\right)=\xi_{\mu[H]}=\Phi_{\pi^{\prime \prime}, \sigma}\left(\xi_{\mu[e]}\right)$. Значит схема $\pi^{\prime \prime}$ на функций разметки $\mu$ строит о. ц. $h \in[H]$ и $F_{\pi^{\prime \prime}}(\mu) \tau H$.

Замечание 1. При построении семантики $\sigma$ и доказательстве отношения

$$
\pi^{\prime} \simeq_{\sigma} \pi^{\prime \prime} \Rightarrow \pi^{\prime \prime} \simeq_{\tau, L} \pi^{\prime \prime}
$$

были использованы только правоконгруэнтные свойства отношения эквивалентности о. ц. $\tau$.

В более общем случае, когда отношение эквивалентности $\tau$ обладает только лишь свойствами правой конгруэнции, утверждение теоремы 9 будет ослаблено.

Следствие 7. Если $\tau$ - правая конгруэниия на $S^{*}$, а множество функция размет$\kappa и L$ согласовано с $\tau$ и замкнуто относителъно операторного сдвига, то существуют тотальные абстрактные семантики $\sigma$ u $\sigma^{\prime}$ такие, что $\sigma^{\prime} \leqslant K(\tau, L) \leqslant \sigma u$ $\sigma^{\prime}$ - гомоморфнъй образ $\sigma$. 
Доказательство. В качестве $\sigma$ возьмем абстрактную семантику $\langle\Sigma, \Sigma, I\rangle$, сопоставленную $K(\tau, L)$ при доказательстве теоремы 9. Согласно приведенному замечанию $K(\tau, L) \leqslant \sigma$. В качестве $\sigma^{\prime}$ возьмем семантику $\left\langle\Sigma^{\prime}, \Sigma^{\prime}, I^{\prime}\right\rangle$, где

$$
\begin{aligned}
\Sigma^{\prime} & =\left\{\xi_{\mu_{h}}: \mu \in L, h \in S^{*}\right\}, \\
I^{\prime} s\left(\xi_{\mu}\right) & =\xi_{\mu_{s}} \text { для каждого } s \in S \text { и } \xi_{\mu} \in \Sigma^{\prime}, \\
I^{\prime} p\left(\xi_{\mu}\right) & =\mu e(p) \text { для каждого } p \in P \text { и } \xi_{\mu} \in \Sigma^{\prime} .
\end{aligned}
$$

Нетрудно видеть, что для любой схемы $\pi$ и функции разметки $\mu \in L$ трасса вычисления программы $\pi$ в семантике $\sigma^{\prime}$ на начальном состоянии $\xi_{\mu}$ совпадает с вычислительной трассой схемы $\pi$ на функции разметки $\mu$. Поскольку функции разметки из $L$ согласованы с отношением эквивалентности $\tau$, для любой $\mu \in L$ из $F_{\pi}(\mu)=h^{\prime} \tau h^{\prime \prime}=F_{\pi}(\mu)$ следует $\Phi_{\pi^{\prime}, \sigma}\left(\xi_{\mu}\right)=\xi_{\mu_{h^{\prime}}}=\xi_{\mu_{h^{\prime \prime}}}=\Phi_{\pi^{\prime \prime}, \sigma}\left(\xi_{\mu}\right)$. Отсюда, учитывая замкнутость $L$ относительно операторного сдвига, получаем $\sigma^{\prime} \leqslant K(\tau, L)$.

Остается отметить, что отображение $\rho$, сопоставляющее каждому состоянию памяти $\xi_{\mu[h]} \in \Sigma$ элемент $\xi_{\mu_{h}} \in \Sigma^{\prime}$, гомоморфно переводит $\sigma$ в $\sigma^{\prime}$.

Как и в случае правоконгруэнтных моделей, естественно задаться вопросом, наскольку характеристические свойства полугрупповых моделей присущи наилучшим аппроксимациям тотальных абстрактных семантик. Из теоремы 3 и предложения 9 следует, что отношение эквивалентности $\tau$ наилучшего приближения $K(\tau, L)$ тотальной семантики $\sigma$ с необходимостью должно быть конгруэнцией на $S^{*}$. Свойство замкнутости множества функций разметки относительно операторного сдвига, напротив, не является неизбежным атрибутом наилучшей или минимальной аппроксимации тотальной семантики. Как показано в [23], отмеченный выше эффект существования моделей $K(\tau, L)$ и $K\left(\tau^{\prime}, L^{\prime}\right)$ таких, что $K\left(\tau^{\prime}, L^{\prime}\right)<K(\tau, L)$ и $L \subset L^{\prime}$, следствием которого, по-существу, является лемма 1, наблюдается и в классе полугрупповых моделей. Поэтому нетрудно построить, подобно тому, как это было сделано при доказательстве теоремы 6 , тотальную семантику $\sigma$, наилучшее приближение которой получается из стандартной аппроксимации $K(\tau(\sigma), L(\sigma))$ путем удаления некоторой функции разметки $\widehat{\mu}=\mu_{h}, \mu \in L(\sigma)$, что приводит к нарушению свойства замкнутости множества функций разметки относительно операторного сдвига.

\section{Список литературы}

1. Rice H. G. Classes of recursively enumerable sets and their decision problems. Trans. Amer. Math. Soc. (1953) 74, №2, 358-366.

2. Подловченко Р. И. Модели последовательностных программ, применяемых для изучения функциональной эквивалентности программ. Кибернетика (1979) №1, 20-28.

3. Подловченко Р. И. Иерархия моделей программ. Программирование (1981) №2, 3-14.

4. Подловченко Р. И. Полугрупповые модели программ. Программирование (1981) №4, 3-13.

5. Глушков В. М., Летичевский А. А. Теория дискретных преобразователей. В сб.: Избранные вопросы алгебры и логики. Наука, Новосибирск, 1973, 5-39.

6. Подловченко Р. И. Модели программ над структурированным базисом. Программирование (1982) №1, 9-19. 
7. Ершов А. П. Современное состояние теории схем программ. Проблемы кибернетики (1973) 27, 87-110.

8. Подловченко Р. И. О проблеме эквивалентных преобразований программ. Программирование. (1986) №6, 3-13.

9. Котов В. Е., Саберфельд В. К. Введение в теорию схем программ. Наука, Новосибирск, 1992.

10. Ляпунов А. А., Яблонский С. В. Теоретические проблемы кибернетики. Проблемъ кибернетики (1963) 27, 7-25.

11. Захаров В. А. Автоматные модели программ. Докл. АН СССР (1989) 309, №1, 24-27

12. Подловченко Р. И., Аланакян Н. А. Регулярные модели программ. Программирование. (1993) №4, 3-12.

13. Янов Ю. И. О логических схемах алгоритмов. Проблемы кибернетики (1958) 1, 75-127.

14. Ершов А. П. Об операторных схемах Янова. Проблемъ кибернетики (1967) 20, 181-200.

15. Подловченко Р. И. Исследование $s$-моделей программ с позиции построения для них алгоритма канонизации. Программирование (1986) №2, 3-13.

16. Подловченко Р. И. Проблема эквивалентности в коммутативных $s$-моделях. Программирование (1987) №5, 6-17.

17. Подловченко Р. И. Схемы программ с монотонными операторами. Программирование (1988) №6, 10-21.

18. Подловченко Р. И. Разрешимость эквивалентности в множестве схем программ с монотонными и частично-перестановочными операторами. Программирование (1990) №5, $3-12$.

19. Тайцлин М. А. Эквивалентность автоматов относительно коммутативной полугруппы. Алгебра и логика (1969) 8, №5, 553-600.

20. Lewis H. R. A new decidable problem with applications. In: Proc. 18 Ann. Symp. on Foundations of Computer Sci. 1979, 62-73.

21. Летичевский А. А. Эквивалентность автоматов относительно полугрупп. Теоретическая кибернетика (1970) №6, 2-45.

22. Лакхем Д., Парк Д. М., Патерсон М. С. О формализованных машинных программах. Кибернетический сборник (1975) 12, 78-114.

23. Захаров В. А. Об одном критерии сравнимости операторных формальных моделей программ. Программирование (1993) №4, 12-26.

24. Захаров В. А. Условия сглаживаемости операторных формальных моделей программ. Программирование (1994) №5, 23-39. 